\title{
DIVERSIDADE E IMPORTÂNCIA DAS ESPÉCIES DE BRIÓFITAS NA CONSERVAÇão dOS ECOSSISTEMAS DO ESTADO do Rio DE JANEIRO
}

\author{
Denise Pinheiro da Costa ${ }^{1}$, Caio A. A. Imbassahy ${ }^{2}$ \& Victor Paulo A. V. da Silva ${ }^{2}$
}

\begin{abstract}
Resumo
(Diversidade e importância das espécies de briófitas na conservação dos ecossistemas do estado do Rio de Janeiro) Este trabalho representa uma contribuição ao "Projeto Flora do Estado do Rio de Janeiro" e objetiva: apresentar lista de táxons de briófitas para o estado; elaborar diagnóstico da diversidade e importância destes na conservação dos ecossistemas; criar banco de dados para os táxons. No total são reconhecidos 1.039 táxons, em 308 gêneros e 95 famílias de briófitas para o estado, das quais 11 são novas ocorrências. As 10 famílias com maior riqueza compreendem $50 \%$ do total de táxons. Para as hepáticas, predomina o padrão neotropical e, para os musgos, o endêmico do Brasil. Em relação à variação altitudinal, as hepáticas ocorrem desde a terra baixa até a montana, enquanto os musgos predominam nas faixas montana e altomontana. Dos 91 municípios levantados apenas 34 apresentam registros de briófitas. Quanto ao status de conservação, 125 táxons são consideradas vulneráveis (VU), 25 ameaçadas (EN) e 147 com dados deficientes (DD), as restantes incluídas na categoria de baixo risco (LR). Das 38 Unidades de Conservação do estado, nove são consideradas prioritárias para levantamentos florísticos de briófitas e 13 importantes centros de diversidade no estado. Dezoito áreas são indicadas como prioritárias para a implantação de novas Unidades de Conservação ou ampliação das já existentes.
\end{abstract}

Palavras-chave: Briófitas, conservação, estado do Rio de Janeiro, Brasil.

\section{Abstract}

(Diversity and importance of the bryophyte taxa in the conservation of the ecosystems of the Rio de Janeiro state) This work is a contribution to the "Projeto Flora do Estado do Rio de Janeiro" and its objectives are: to present a cheklist of the bryophyte taxa to the state; to make a diagnosis of the diversity and importance of the bryophyte taxa in the conservation of the ecosystems; to create a data base for the bryophyte taxa. In total are recognized 1,039 taxa in 308 genera and 95 families of bryophytes to the state, of which 11 are new records. The 10 largest families account for $50 \%$ of the total diversity. For the hepatics, the neotropical distribution pattern predominate and for the mosses, the endemic to Brazil. In relation to the altitude variation, the hepatics show the highest diversity in the lowland to montane areas, while the mosses in the montane and uppermontane ones. There are 91 counties in the state, and only 34 present records of the bryophytes. One hundred and twenty five taxa are considered vulnerable (VU), 25 endangered (EN) and 147 with deficient data (DD), the remains had been enclosed in the low risk (LR). There are 38 Units of Conservation in the state, 9 are considered priorities for floristic surveys of bryophytes and 13 are important centers of diversity in the state. Eighteen areas are indicated as priorities for implantation of new Conservation Units or enlargment of already the existing ones.

Key-words: Bryophytes, conservation, Rio de Janeiro state, Brazil.

\section{INTRODUÇÃo}

Brasil, México, Colômbia e Indonésia são considerados países detentores da megadiversidade. Estima-se que o Brasil abrigue entre 15$20 \%$ de cerca de um milhão e meio das espécies do planeta (microorganismos a angiospermas e mamíferos). É o país com a maior diversidade de angiospermas (20-22\% das 50-56 mil espécies), o segundo em número de espécies de mamíferos (10\% das 525 espécies) e anfíbios (10\% das 520 espécies), e o terceiro em aves (17\% de 1670 espécies), (Projeto Flora do
Estado do Rio de Janeiro 2002). Em relação às briófitas, o Brasil apresenta ca. 18\% (Yano 1996a) das 18000 espécies ocorrentes no mundo (Shaw \& Goffinet 2000).

Estudos recentes afirmam que os valores da diversidade biológica brasileira e dos serviços dela oriundos situam-se na casa dos trilhões de dólares anuais, assim, a prospecção da diversidade biológica é um componente relevante na estratégia de desenvolvimento econômico do país e do estado (Projeto Flora do Estado do Rio de Janeiro 2002).

Artigo recebido em 08/2004. Aceito para publicação em 02/2005

${ }^{1}$ Pesquisadora - Instituto de Pesquisas Jardim Botânico do Rio de Janeiro, Programa Diversidade Taxonômica, Rua Pacheco Leão 915, 22460-030, Rio de Janeiro, RJ, dcosta@ jbrj.gov.br

${ }^{2}$ Bolsistas de Iniciação Científica - Instituto de Pesquisas Jardim Botânico do Rio de Janeiro (PIBIC/CNPq). 
Dada a sua localização e imensa diversidade de formações geográficas, estendendo-se da montanha ao mar, o estado do Rio de Janeiro, caracteriza-se não só por uma significativa diversidade biológica, como também pelo alto grau de endemismos, demonstrando a importância da sua flora e fauna, sendo considerado centro de diversidade para espécies da mata atlântica (Projeto Flora do Estado do Rio de Janeiro 2002).

Esta diversidade biológica relaciona-se intimamente com a grande variedade de habitats existentes no estado, desde os campos de altitude (Itatiaia), descendo pela montanha com a Mata Atlântica (altomontana, montana e terra baixa), passando pelas restingas com formações florestais, inundadas ou não, e formações arbustivas, nas quais se inserem cerca de 60 lagoas ao longo do litoral (doces, salinas e hipersalinas), chegando aos prados salinos, manguezais e praias (Projeto Flora do Estado do Rio de Janeiro 2002).

É urgente que o estado do Rio de Janeiro tenha sua flora o mais completamente conhecida e disponível para a comunidade científica e para a sociedade, em particular, os tomadores de decisão, com vistas a um manejo adequado do seu imenso patrimônio natural. A elaboração de uma flora não só contribui para a identificação de plantas, determinando quais nomes podem ser usados e informando sobre caracteres morfológicos, distribuição e habitats de espécies, como também subsidia o gerenciamento ambiental na administração de Unidades de Conservação, servindo como base para estudos de prospecção no que diz respeito a seus usos potenciais pela sociedade (Projeto Flora do Estado do Rio de Janeiro 2002).

Grande parte do conhecimento dos táxons de musgos do Brasil, ainda se restringe aos catálogos de Yano (1981, 1989, 1995, 1996a), enquanto para as hepáticas e antóceros, recentemente foi elaborada uma flora por Gradstein \& Costa (2003). O estado do Rio de Janeiro conta com uma brioflora rica, porém este conhecimento encontra-se disperso em poucas publicações e flórulas, não existindo uma lista de táxons de briófitas para o estado, tampouco um diagnóstico ambiental com os táxons de briófitas. Os objetivos deste trabalho são: contribuir com o "Projeto Flora do Estado do Rio de Janeiro" gerando uma lista de táxons de briófitas e elaborando um diagnóstico da diversidade e importância destes na conservação dos ecossistemas e gerar um banco de dados de táxons para o estado.

\section{Histórico}

A flora de briófitas do Rio de Janeiro é considerada bem conhecida quando comparada a outros estados do país. Os trabalhos clássicos que incluem a brioflora do Rio de Janeiro foram realizados por Hornschuch (1840), Hampe (1870, 1872, 1874a, 1874b, 1877, 1879), Müller (1898, 1900, 1901), Stephani (1905-1912), Dusén (1903), Brotherus (1924) e Herzog (1925), que basearam-se em coleções históricas feitas por Glaziou, Hampe, Ule, entre outros, no século XIX.

Após 1925 pouco foi publicado a respeito da brioflora do estado do Rio de Janeiro, até que, a partir de 1988, diversos trabalhos foram realizados por Costa $(1988,1992,1994)$, Costa \& Yano (1988, 1995), Oliveira e Silva (1998), Molinaro \& Costa (2001), entre outros. Yano $(1981,1984,1989,1995)$ sumarizou o conhecimento das espécies de briófitas do Brasil, e a informação contida em seus catálogos tornouse uma obra referencial para a briologia no país, assim como a recente flora de hepáticas realizada por Gradstein \& Costa (2003).

Até o presente, o único diagnóstico realizado com táxons de briófitas para um estado do Brasil, é o de Pôrto \& Germano (2002), para Pernambuco.

\section{Material e Métodos \\ Lista de táxons}

Objetivando realizar um diagnóstico da importância e diversidade de briófitas do estado do Rio de Janeiro, foi elaborada uma lista preliminar com os táxons de musgos citados para o estado com base nos catálogos de Yano (1981, 1989, 1995, 1996a), com adições de publicações mais recentes e das coleções dos herbários do Instituto de Pesquisas Jardim 
Botânico do Rio de Janeiro (RB) e da Universidade do Estado do Rio de Janeiro (HRJ). Para os táxons de hepáticas e antóceros, a lista foi baseada na recente flora elaborada por Gradstein \& Costa (2003) para o Brasil. Os dados deste trabalho foram obtidos na bibliografia disponível até $\mathrm{o}$ ano de 2003.

A atualização nomenclatural da lista de táxons de musgos foi baseada, principalmente, nos trabalhos de Zander (1993), Sharp et al. (1994), Churchill \& Linares (1995a,b), Delgadillo et al. (1995), Florschütz-de-Waard (1996), Buck (1998), Crosby et al. (1999), revisões da Flora Neotropica (Buck \& Ireland 1989, Frahm 1991, Reese 1993, Ireland \& Buck 1994, Hedenäs 2003), obras específicas para famílias e gêneros, como Schültze-Motel (1970), Zander (1972), Buck (1979), Ochi (1980, 1981a,b; 1982), Allen (1987), Fife (1987), Sastre-de-Jesus (1987), Tixier (1988), Crum (1990a,b,c; 1992 , 1993), Pursell (1994), Spence (1996), Frahm (1996, 1997), LaFarge-England (1998), Muñoz (1999), Reiner-Drehwald \& Goda (2000), Heinrichs et al. (2000), no banco de dados W'MOST (http://mobot.mobot.org/W3T/Search/ most.html), e em consulta a especialistas. Para as hepáticas e antóceros, aceitou-se os dados contidos em Gradstein \& Costa (2003). As classificações adotadas seguem aquelas encontradas em Shaw \& Goffinet (2000).

\section{Organização dos dados}

Os dados foram organizados em uma tabela com as seguintes informações para cada táxon: família, gênero, espécie, autor, municípios de ocorrência, distribuição no Brasil por estado e no mundo, variação altitudinal no Brasil, status de conservação. A análise dos dados gerou três tipos de resultados: 1) uma lista com a distribuição dos táxons de musgos, hepáticas e antóceros do estado do Rio de Janeiro (Costa et al. 2005); 2) uma análise do status de conservação dos táxons de briófitas do estado do Rio de Janeiro (Costa et al. inédito); 3) um diagnóstico sobre a diversidade e importância dos táxons de briófitas na conservação dos ecossistemas do estado do Rio de Janeiro (esta publicação).
As ocorrências para os municípios do estado do Rio de Janeiro foram extraídas dos catálogos de Yano (1981, 1989, 1995), das obras originais, dos trabalhos mais recentes de Costa \& Yano (1995, 1998), Oliveira e Silva (1998), Molinaro \& Costa (2001), Oliveira e Silva $e t$ al. (2002), Gradstein \& Costa (2003), Costa \& Lima (2005) e de coleções dos herbários RB e HRJ. Para as ocorrências nos demais estados do país, além dos trabalhos supracitados, foram consultadas as dissertações e teses de Sá (1995), Castro (1997), Santiago (1997), Oliveira e Silva (1998), Visnadi (1998), Bastos (1999), LemosMichel (1999), Câmara (2002), Germano (2003); os trabalhos de Sehnem (1969, 1970, 1972, 1976, 1978, 1979, 1980), Schäfer-Verwimp \& Vital (1989), Schäfer-Verwimp (1989, 1991, 1992, 1996), Schäfer-Verwimp \& Giancotti (1993), Vital \& Visnadi (1994), Lisboa \& IlkiuBorges (1995, 1997, 2001), Pôrto \& Bezerra (1996), Yano (1996b), Yano \& Oliveira e Silva (1997), Churchill (1998), Oliveira e Silva \& Yano (1998), Bastos \& Villas-Bôas-Bastos (1998), Villas-Bôas-Bastos \& Bastos (1998), Lisboa et al. (1999), Yano \& Mello (1999), Bastos et al. (1998a,b, 2000), Visnadi \& Vital (2000), Yano \& Colletes (2000), Yano \& Costa (2000), Visnadi \& Vital (2000, 2001), Pôrto \& Germano (2002), Costa (2003), Costa \& Silva (2003), Santos \& Lisboa (2003), Gradstein \& Costa (2003). A distribuição no mundo e a variação altitudinal no país, foram baseadas nos dados da literatura disponíveis para cada táxon.

\section{Análise da variação altitudinal}

A classificação da vegetação adotada é a de Veloso et al. (1991), onde floresta de terra baixa $=0-200 \mathrm{~m}$; floresta submontana $=200$ $500 \mathrm{~m}$; floresta montana $=500-1.500 \mathrm{~m}$; e floresta altomontana $=>1.500 \mathrm{~m}$.

\section{Mapa da riqueza de espécies por município}

Com a finalidade de analisar a riqueza da brioflora por municípios e unidades de conservação no estado, os dados de distribuição de cada táxon foram plotados em um mapa do estado do Rio de Janeiro. 


\section{Análise do status de conservação dos táxons}

A caracterização do status de conservação dos táxons de briófitas do estado do Rio de Janeiro foi baseada nas diretrizes propostas pelo grupo de especialistas em briófitas IUCN SSC (Hallingbäck et al. 1996; Hallingbäck \& Hodgetts 2000), e complementada com os primeiros trabalhos que contemplaram este tipo de análise no país, realizados por Costa (1999) e Pôrto \& Germano (2002).

\section{Seleção de áreas prioritárias}

O mapa de distribuição dos táxons por município foi comparado com os mapas de vegetação e de unidades de conservação do estado (Atlas das unidades de conservação da natureza do estado do Rio de Janeiro 2001; SOS Mata Atlântica/INPE 2002), com a finalidade de apontar áreas para futuros levantamentos da brioflora; identificar os centros de diversidade; indicar áreas a serem conservadas por meio de novas Unidades de Conservação e reforçar a importância das Unidades de Conservação existentes.

\section{Resultados e discussão \\ Composição florística}

No total, são reconhecidos para o estado do Rio de Janeiro 1039 táxons, distribuídos em 308 gêneros e 95 famílias, sendo 5 de antóceros (2 famílias e 3 gêneros), 333 de hepáticas (30 famílias e 100 gêneros), e 701 de musgos (63 famílias e 205 gêneros). A brioflora do estado é considerada rica, apresentando $33 \%$ do total de táxons do país, $26 \%$ do neotrópico e $6 \%$ do mundo (Tabela 1).

Entre as 95 famílias de briófitas ocorrentes no estado, 10 apresentaram maior riqueza específica, a saber: Lejeuneaceae, Dicranaceae, Pilotrichaceae, Orthotrichaceae, Sematophyllaceae, Sphagnaceae, Pottiaceae, Hypnaceae, Bryaceae e Brachyteciaceae, totalizando 50\% das espécies do estado. Segundo Gradstein \& Pócs (1989), estas famílias, com exceção de Sphagnaceae, Pottiaceae e Bryaceae, estão entre as 15 principais famílias encontradas em inventários florísticos no neotrópico (Figura 1).
Tabela 1 - Comparação do número de táxons de briófitas do estado do Rio de Janeiro com outras regiões.

\begin{tabular}{lcl}
\hline Regiões & \multicolumn{1}{c}{ Número } & \multicolumn{1}{c}{ Referência } \\
de espécies & \\
\hline Rio de Janeiro & 1.039 & Esta publicação \\
Brasil & 3.200 & Costa \& Pôrto (2003) \\
Neotrópico & 4.000 & Gradstein et al. (2001) \\
Mundo & 18.000 & Shaw \& Goffinet (2000) \\
\hline
\end{tabular}

Com relação ao padrão de distribuição, predominaram o neotropical e o endêmico do Brasil, abrangendo $57 \%$ dos táxons. O restante dos táxons são cosmopolitas, pantropicais, afroamericanos ou ocorrem na América tropical e subtropical (Figura 2). Estes resultados demonstram um expressivo número de táxons endêmicos para o país que, de uma maneira geral, ocorrem no ecossistema mata atlântica.

Em relação à distribuição dos táxons, 228 estão amplamente distribuídos no país, ocorrendo em quatro ou cinco regiões geográficas; 261 distribuem-se de forma descontínua, possivelmente por falta de estudos e coletas; 260 ocorrem apenas nas Regiões Sul e Sudeste; e 290 exclusivos da Região Sudeste, dos quais 161 são exclusivos do estado do Rio de Janeiro, sendo 90 endêmicos, confirmando assim a Região Sudeste como um dos centros de diversidade do país (Figura 3).

De acordo com estes resultados, o Rio de Janeiro apresenta uma expressiva contribuição para a brioflora do país, relacionada, principalmente, com as diferentes formações do ecossistema mata atlântica que ocorrem no estado. O município de Itatiaia apresenta o maior número de táxons endêmicos, seguido pelos municípios que pertencem à Serra dos Órgãos, principalmente, Teresópolis e Petrópolis (Figura 4).

A análise da distribuição altitudinal dos táxons no país, demonstrou que a mais alta diversidade, em termos de números de táxons, ocorre na faixa montana, entre 500-1.500 m (Tabela 2). Esta faixa apresentou 822 táxons, dos quais 117 são exclusivos, não ocorrendo nas demais faixas. Foram encontradas na terra baixa (0$200 \mathrm{~m}) 598$ táxons, na submontana (200-500 m) 590 e na altomontana (acima de $1.500 \mathrm{~m}$ ) 442. A faixa altomontana apresentou 97 táxons 


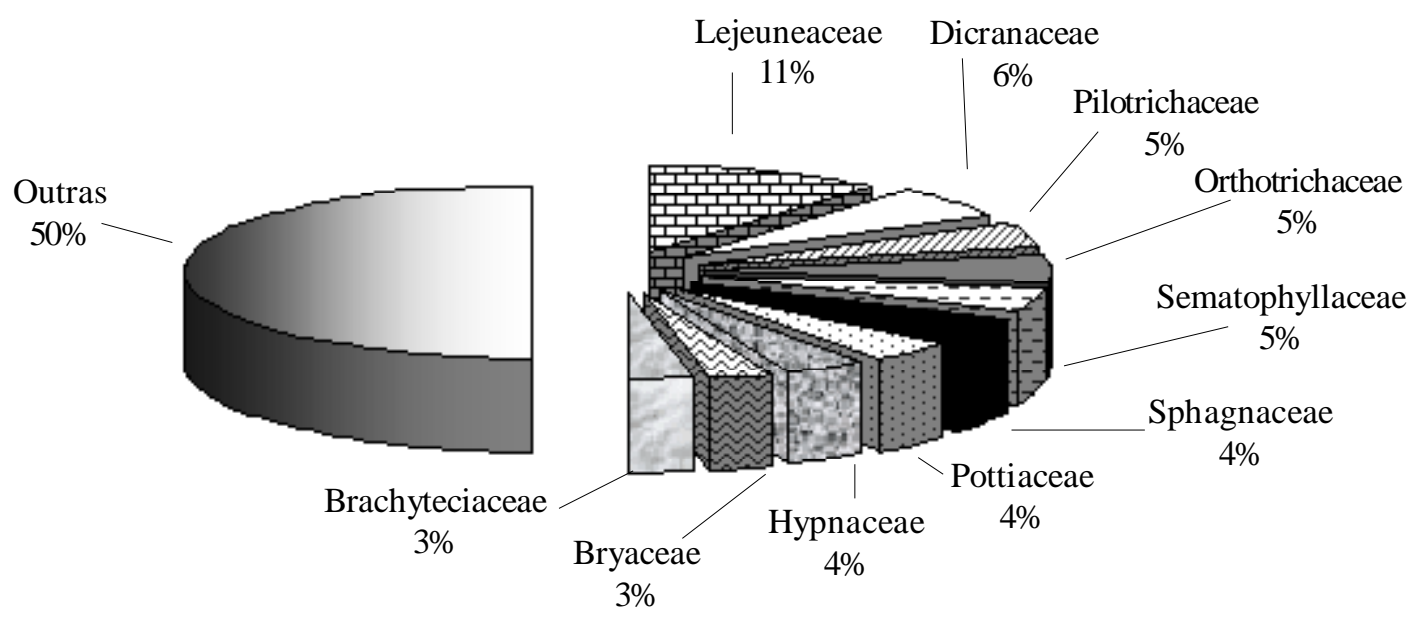

Figura 1 - Principais famílias de briófitas ocorrentes no estado do Rio de Janeiro.

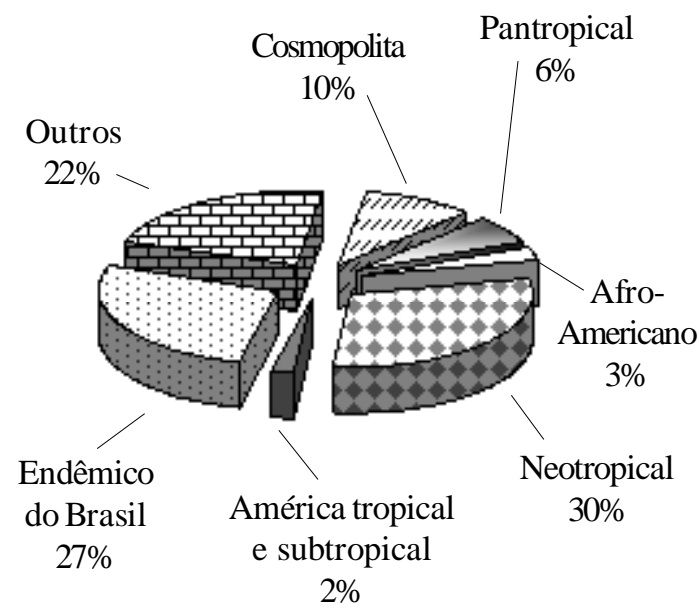

Figura 2 - Padrão de distribuição no mundo das espécies de briófitas do estado do Rio de Janeiro.

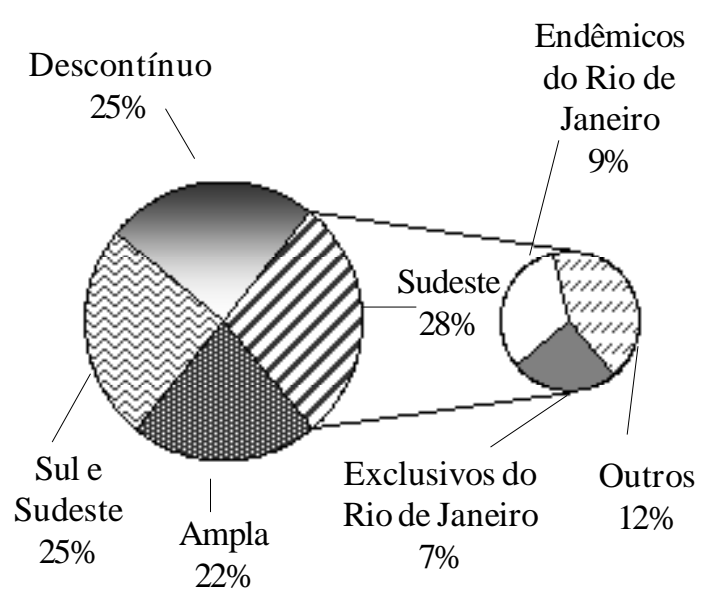

Figura 3 - Padrão de distribuição no país dos táxons de briófitas do estado do Rio de Janeiro.

Rodriguésia 56 (87): 13-49. 2005

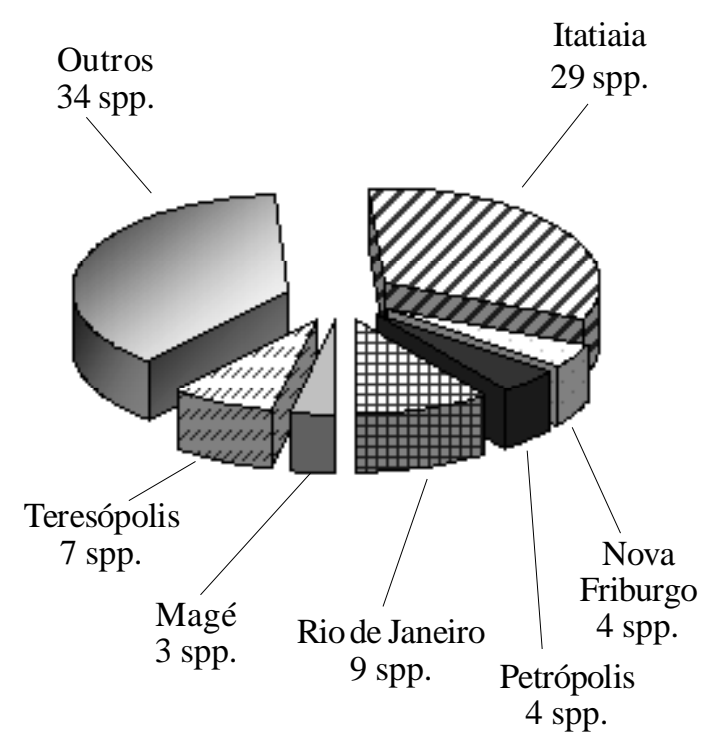

Figura 4 - Distribuição por município de ocorrência dos táxons endêmicos do estado do Rio de Janeiro.

Tabela 2 - Distribuição altitudinal dos táxons de briófitas do estado do Rio de Janeiro.

\begin{tabular}{lcc}
\hline $\begin{array}{c}\text { Faixa } \\
\text { altitudinal }\end{array}$ & $\begin{array}{c}\text { Número } \\
\text { de táxons }\end{array}$ & $\begin{array}{c}\text { Táxons restritos } \\
\text { à faixa }\end{array}$ \\
\hline $0-200 \mathrm{~m}$ & 598 & 40 \\
$200-500 \mathrm{~m}$ & 590 & 2 \\
$500-1.500 \mathrm{~m}$ & 822 & 117 \\
$>1.500 \mathrm{~m}$ & 442 & 97 \\
\hline
\end{tabular}


restritos a ela, indicando a grande importância ecológica desta faixa, já que em poucas regiões do Brasil encontram-se altitudes superiores a $1.500 \mathrm{~m}$. O elevado número de táxons encontrados na terra baixa, reflete a grande extensão desta área no país. Resultados similares foram encontrados por Gradstein (1995), Uribe \& Gradstein (1999), Pôrto \& Germano (2002), Gradstein \& Costa (2003) e Costa \& Lima (2005), para o país ou para a América do Sul.

Para avaliar a riqueza brioflorística por município, foram plotados num mapa do estado do Rio de Janeiro o número total de táxons por município. Dos 91 municípios, apenas 34 apresentam registros de táxons de briófitas, demonstrando a carência de estudos com a brioflora em grande parte do estado (Figura 5).

Analisando o mapa de riqueza de táxons por município (Figura 5), observa-se maior número de táxons em Itatiaia (489 spp), Rio de Janeiro (346 spp), Nova Friburgo (282 spp), Teresópolis (272 spp), Niterói (227 spp), Angra dos Reis (210 spp), Petrópolis (158 spp), Mangaratiba (139 spp) e Parati (117 spp). A região conhecida como Costa Verde, que engloba os municípios de Angra dos Reis, Mangaratiba e Parati, apresenta a maior relação de área verde para área urbana no estado, tendo sido estudada por Oliveira e Silva (1998) e Costa (1997). Os municípios de Itatiaia, Teresópolis, Petrópolis e Nova Friburgo, são importantes áreas de mata atlântica montana e altomontana do estado, bem preservadas, tendo sido estudadas desde o final do século XIX até o início do século XX, com exceção de Nova Friburgo. Recentemente alguns destes municípios vêm sendo estudados por Costa (1995), Costa \& Lima (2005). O município do Rio de Janeiro apresenta coleções históricas como as realizadas por Ule, Glaziou, entre outros, consideradas importantes e de referência. Em relação ao município de Niterói, as coletas realizadas por Oliveira e Silva (HRJ) comprovaram a riqueza da sua brioflora.

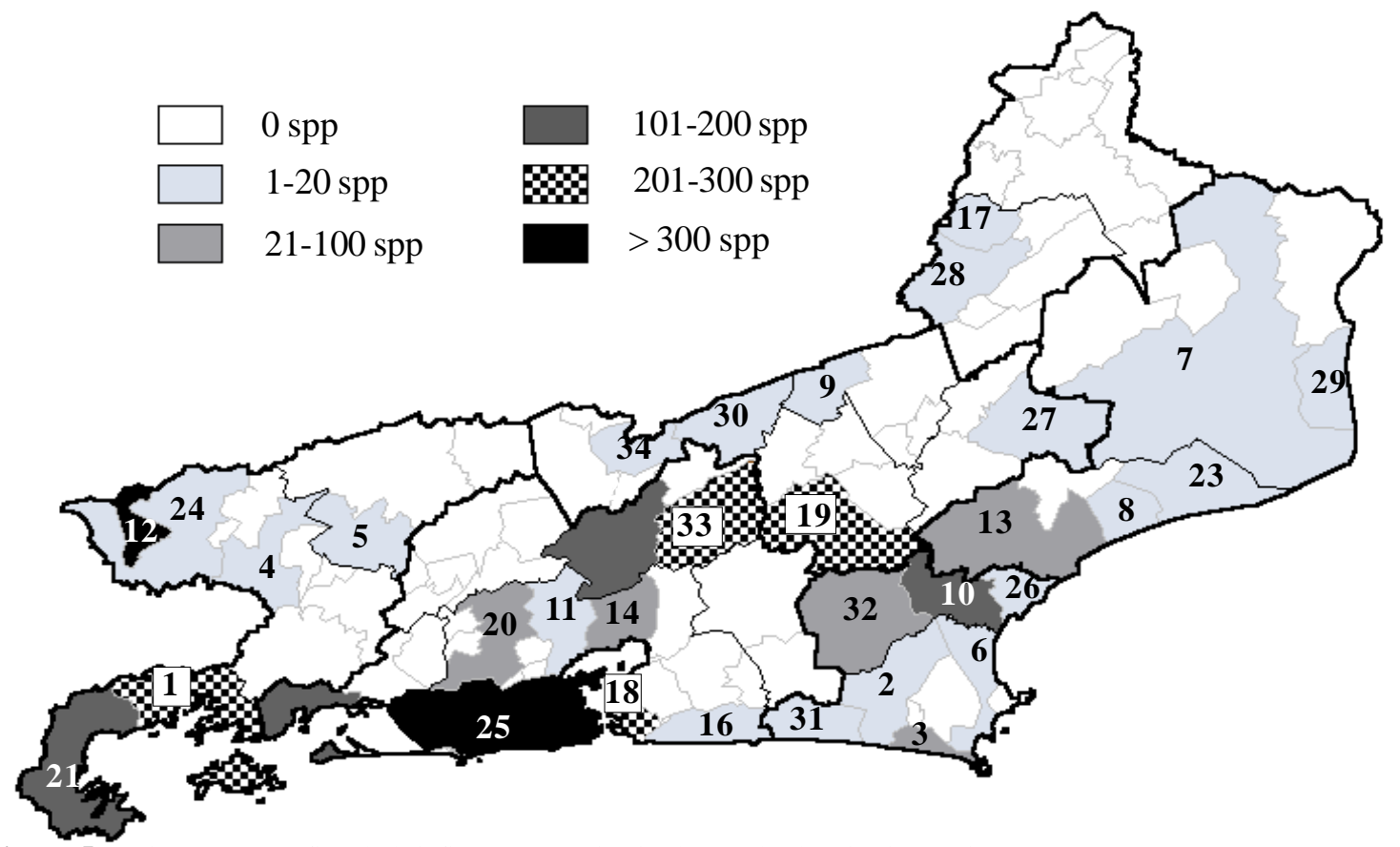

Figura 5 - Riqueza específica de briófitas por município do estado do Rio de Janeiro.

1 = Angra dos Reis (210); 2 = Araruama (8); 3 = Arraial do Cabo (22); 4 = Barra Mansa (1); 5 = Barra do Piraí (1); 6 = Cabo Frio (12); 7 = Campos dos Goytacazes (1); 8 = Carapebus (5); 9 = Carmo (1); 10 = Casimiro de Abreu (109); 11 = Duque de Caxias (1); 12 = Itatiaia (489); 13 = Macaé (49); 14 = Magé (87); 15 = Mangaratiba (139); 16 = Maricá (17); 17 = Miracema (1); 18 = Niterói (227); 19 = Nova Friburgo (282); 20 = Nova Iguaçu (92); 21 = Parati (117); $22=$ Petrópolis (158); 23 = Quissamã (10); 24 = Resende (17); 25 = Rio de Janeiro (346); 26 = Rio das Ostras (12); 27 = Santa Maria Madalena (1); 28 = Santo Antônio de Pádua (1); 29 = São João da Barra (4); 30 = Sapucaia (1); 31 = Saquarema (14); 32 = Silva Jardim (45); 33 = Teresópolis (272); 34 = Três Rios (1). 


\section{Conservação}

Seguindo os critérios da IUCN/SSC Bryophyte Specialist Group (Hallingbäck et al. 1996, Hallingbäck \& Hodgetts 2000), entre os 1.039 táxons ocorrentes no estado do Rio de Janeiro, 125 foram considerados vulneráveis (VU), 25 ameaçados (EN) e 147 com dados deficientes (DD), os restantes incluídos na categoria de baixo risco (LR). A análise do status de conservação dos táxons aqui realizada restringiu-se ao estado.

Das 38 Unidades de Conservação do estado (Atlas das unidades de conservação da natureza do estado do Rio de Janeiro 2001), nove têm sua brioflora praticamente desconhecida, sendo consideradas prioritárias para futuros levantamentos florísticos: APA da Bacia do Frade, APA de Gericinó-Mendanha, APA de Guapimirim, APA da Serra de Sapiatiba, ARIE da Floresta da Cicuta, ESEC do Paraíso, FLONA Mário Xavier, PE do Desengano, REBIO União, e 13 foram consideradas importantes centros de diversidade do estado: APA da Floresta do Jacarandá, APA de Mangaratiba, APA de Tamoios, ESEC de Tamoios, PARNA do Itatiaia, PARNA da Serra da Bocaina, PARNA da Serra dos Órgãos, PARNA da Tijuca, PE da Ilha Grande, PE da Serra de Tiririca, REBIO de Araras, REBIO da Praia do Sul e RESEC de Alcobaça.

Com base na representatividade das fomações vegetais e ecossistemas, em termos de tamanho e estado de conservação, 18 áreas são indicadas como prioritárias para a implantação de novas unidades de conservação ou para ampliação das unidades já existentes. Em relação àquelas unidades que devem ser ampliadas estão: Cachoeiras de Macacu (ESEC do Paraíso, floresta de terra baixa a montana) e Silva Jardim (REBIO Poço das Antas, floresta de terra baixa). As áreas sugeridas para a implantação de novas Unidades de Conservação são: Barra do Piraí/Valença/Vassouras (floresta montana), Bom Jardim/Trajano de Morais (floresta montana), Bom Jesus do Itabapoana (floresta de terra baixa), Cambuci (floresta de terra baixa), Campos (floresta de terra baixa), Itaperuna/Natividade (floresta de terra baixa), Miracema/Santo Antônio de Pádua (floresta de terra baixa), Natividade/Porciúncula/VarreSai (floresta de terra baixa à montana), Nova Friburgo (floresta montana a altomontana), Piraí (floresta submontana), Rio Bonito (floresta de terra baixa), Rio Claro (floresta submontana a montana), São Francisco do Itabapoana (floresta de terra baixa), São João da Barra (floresta de terra baixa), Cantagalo/Cordeiro/ Carmo/Duas Barras (floresta de terra baixa), Sumidouro/Sapucaia (floresta de terra baixa).

\section{Conclusões}

A brioflora do estado do Rio de Janeiro é considerada rica, apresentando $33 \%$ do total de táxons do país e, com base nos resultados aqui apresentados, fica claro que o seu conhecimento ainda éincompleto. Apesar da quantidade de dados recentes, a brioflora nos diferentes municípios ainda é pouco conhecida. Os trabalhos realizados no estado concentram-se nas regiões de floresta montana, como Itatiaia e Serra dos Órgãos e nas regiões litorâneas, como Rio de Janeiro e Angra dos Reis, havendo carência de informação nas regiões do norte fluminense, Vale do Paraíba e em diferentes partes da Serra do Mar, onde ainda hoje existem poucos ou nenhum registro de táxons de briófitas.

Apesar da ocorrência de 95 famílias de briófitas no estado, 10 compreendem ca. 50\% do total de táxons, demonstrando que a diversidade da brioflora está concentrada em um pequeno número de famílias, como ocorre na América tropical em geral.

A brioflora do estado do Rio de Janeiro conta com um número expressivo de espécies vulneráveis (VU) e ameaçadas de extinção (EN), (150 spp - 14,4\%), pelo menos ao nível regional. Entre as espécies consideradas de baixo risco (LR), muitas estão na dependência da conservação de seus habitats. Uma grande parte destes táxons estão incluídos na categoria de dados deficientes (DD), dificultando a análise mais aprofundada da brioflora do estado. Muitos táxons que se encontram em áreas legalmente protegidas por unidades de conservação (PARNAs, RESECs etc), ainda são considerados como ameaçados ou vulneráveis, principalmente por ocorrerem habitats restritos e de considerável fragilidade. 


\section{Lista dos táxons de briófitas do estado}

Aqui estão incluídos, em ordem alfabética por divisão, família, gênero e espécie, 1039 táxons de briófitas. Entre parênteses, estão o número de famílias, gêneros e táxons encontradas no estado e, ao lado de cada táxon, a ocorrência nos municípios, a variação altitudinal no país, e o status de conservação do táxon no estado, cujos dados foram retirados de Costa et al. (inédito). A abreviação "s/alt." foi utilizada em casos de altitude indefinida e/ou ausência de dados na literatura.

Abreviaturas para os 34 municípios do estado com registros de táxons, estando entre parênteses o número total de táxons: $\mathrm{ACA}=$ Arraial do Cabo (22); $\mathrm{ANG}=$ Angra dos Reis (210); $\mathrm{ARA}=\operatorname{Araruama}(8)$; $\mathrm{BAM}=$ Barra Mansa (1); BAP = Barra do Piraí (1); $\mathrm{CAB}=$ Casimiro de Abreu (109); $\mathrm{CAF}=$ Cabo Frio (12); $\mathrm{CAM}=\mathrm{Campos}$ dos Goytacazes (1); CAP = Carapebus (5); CAR=Carmo(1); DQC = Duque de Caxias (1); ITA= Itatiaia (489); MAC = Macaé (49); MAG = Magé (87); MAN = Man-garatiba (139); MAR = Maricá (17); MIR = Mira-cema (1); NIT= Niterói (227); NVF= Nova Fri-burgo (282); NVI = Nova Iguaçu (92); PAR = Parati (117); PET= Petrópolis (158); QUI = Quissamã (10); RES = Resende (17); RJN = Rio de Janeiro (346); ROS = Rio das Ostras (12); SAP = Sapucaia (1); SAQ = Saquarema (14); SJB = São João da Barra (4); SMM = Santa Maria Madalena (1); STA = Santo Antônio de Pádua (1); SVJ = Silva Jardim (45); TER = Teresópolis (272); TRR = Três Rios (1); S/L= Sem localidade (89).

\section{Divisão Anthocerotophyta (2/3/5)}

\section{Anthocerotaceae (2/4)}

Anthoceros lamellatus Steph. - RJN; ca. 800 m; EN

Anthoceros punctatus L. - NIT, PAR, RJN, TER; 0-800 m

Phaeoceros laevis subsp. carolinianus (Michx.) Prosk. - PAR; 0-1000 m

Phaeoceros laevis (L.) Prosk. subsp. laevis - ANG, CAB, MAN, NIT, RJN, TER; 0-1250 m; VU

\section{Dendrocerotaceae (1/1)}

Dendroceros crispus (Sw.) Nees - CAB, ITA, TER; 500-1200 m

\section{Divisão Bryophyta (63/205/701)}

\section{Adelotheciaceae (1/1)}

Adelothecium bogotense (Hampe) Mitt. - ITA, NVF, TER; 500-2300 m

Amblystegiaceae (5/5)

Calliergonella cuspidata (Hedw.) Loeske. - RJN; 0-1660 m

Campyliadelphus chrysophyllus (Brid.) Kanda. - RJN; s/alt; DD

Drepanocladus perplicatus (Dusén) G. Roth - ITA; 2140 m; VU

Hygrohypnum laevigatum (Herzog) J.-P. Frahm - PET; 2200 m; EN

Warnstorfia exnnulata (Schimp.) Loeske - ITA; 2300-2400 m; VU

Andreaceae (1/6)

Andreaea microphylla Müll. Hal. - ITA; 2400 m; VU

Andreaea rupestris Hedw. - ITA, TER; 1200-2500 m; VU

Andreaea spurioalpina Müll. Hal. - ITA; 2300-2750 m; VU

Andreaea squarrosofiliformis Müll. Hal. - ITA; 2300 m; VU

Andreaea striata Mitt. - TER; ca. 2000 m; VU

Andreaea subulata Harv. - ITA, TER; 2000-2800 m; VU

Anomodontaceae (1/1)

Herpertineuron toccoae (Sull. \& Lesq.) Cardot - MAG, MAN, NIT; ca. 500 m

\section{Bartramiaceae (4/21)}

Bartramia halleriana Hedw. - NVF; 990-2170 m

Bartramia longifolia Hook. - MAG; >1200 m; EN

Bartramia mathewsii subsp. brasiliensis Fransén - ITA; 2000-2600 m

Breutelia grandis (Hampe) Paris - ITA, NVF, PET, RJN, TER; 500-2000 m

Breutelia microdonta (Mitt.) Broth. - ITA; 1500-2100 m 
Breutelia subdisticha (Hampe) A. Jaeger - ITA, NVF, PAR, PET, RJN, TER; 500-2500 m Breutelia subtomentosa (Hampe) A. Jaeger - ITA, NVF, PAR, RES, TER; 800-2500 m Breutelia wainioi Broth. - ITA, NVF; 500-2500 m

Leiomela bartramioides (Hook.) Paris - S/L; s/alt.; DD

Leiomela piligera (Hampe) Broth. - ITA, MAG, NVF, RJN, TER; 800-2000 m

Philonotis cernua (Wilson) D.G. Griffin \& W.R. Buck - ITA; 900-2500 m

Philonotis gardneri (Müll. Hal.) A. Jaeger - ITA, MAG, NVF, PAR, RJN; 0-2000 m

Philonotis glaucescens (Hornsch.) Broth. - ITA, NVF, PAR, PET, RJN, TER; 0-1600 m

Philonotis gracillima Aongstr. - S/L; 0-1100 m

Philonotis hastata (Duby) Wijk \& Margad. - ANG, CAB, MAN, NIT; 0-600 m

Philonotis humilis Brid. - TER; 0-1200 m

Philonotis pellucidiretis (Müll. Hal.) Paris - ITA; 1350-1800 m; DD

Philonotis rufiflora (Hornsch.) Reichardt - MAG, TER; 0-1200 m

Philonotis sphaerocarpa (Hedw.) Brid. - S/L; 0-900 m

Philonotis spiralis (Hampe) A. Jaeger - S/L; ?-600 m; DD

Philonotis uncinata (Schwägr.) Brid. - ANG, CAB, ITA, MAG, MAN, NIT, NVF, PAR, RJN; 0-1350 m

\section{Brachyteciaceae (10/29)}

Aerolindigia capillacea (Hornsch.) M. Menzel - ANG, ITA, MAN, NIT, NVF, PAR, RJN; 0-1300 m

Brachythecium poadelphus Müll. Hal. - PET; ca. 800 m; VU

Brachythecium ruderale (Brid.) W.R. Buck - ITA; 500-1400 m

Brachythecium sulphureum (Geh. \& Hampe) Paris - PET; 0-800 m

Meteoridium remotifolium (Müll. Hal.) Manuel - ITA, MAG, NVI, NVF, PAR, PET, RJN, TER; 0-2200 m

Palamocladium leskeoides (Hook.) E. Britton - NVF; 0-1100 m

Platyhypnidium aquaticum (A. Jaeger) M. Fleisch. - NVF; 0-900 m

Platyhypnidium intermedium Herzog - TER; ca. 1200 m; DD

Puiggariopsis aurifolia (Mitt.) M. Menzel - RJN; 0-1200 m

Rhynchostegium apophysatum (Hornsch.) A. Jaeger - MAG, TER; 0-1200 m; DD

Rhynchostegium compridense (Broth.) Paris - NVF, RJN; 0-2000 m; DD

Rhynchostegium finitimum (Hampe) Aongstr. - S/L; 0-1100 m; DD

Rhynchostegium megapolitanum (Web. \& Mohr.) B.S.G. - TER; 800-1200 m; VU

Rhynchostegium microthamnioides Müll. Hal. - ITA; 700-1300 m

Rhynchostegium pallidius (Hampe) A. Jaeger - S/L; nível do mar; DD

Rhynchostegium rivale (Hampe) A. Jaeger - ITA, NVF, RJN, TER; 0-2000 m; VU

Rhynchostegium sellowii (Hornsch.) A. Jaeger - ITA, SVJ, TER; 0-2000 m

Squamidium brasiliense (Hornsch.) Broth. - ITA, NVF, PET, RJN, SVJ, TER; 0-2000 m

Squamidium diversicoma (Hampe) Broth. - RJN; 0-700 m

Squamidium isocladum (Renauld \& Cardot) Broth. - NIT, NVF; 500-1200 m

Squamidium leucotrichum (J. Taylor) Broth. - ANG, CAB, ITA, MAN, NIT, NVF, PAR, RJN, SVJ, TER; $0-1600 \mathrm{~m}$

Squamidium macrocarpum (Spruce) Broth. - ITA, PET, RJN, TER; 0-900 m

Squamidium nigricans (Hook.) Broth. - ANG, CAB, MAN, NIT; 0-900 m

Steerecleus beskeanus (Müll. Hal.) H. Robinson - ITA, NVF, RJN, TER; 0-1350 m

Steerecleus scariosus (J. Taylor) H. Robinson - ANG, CAB, MAN, NIT, RJN, TER; 0-800 m

Zelometeorium ambiguum (Hornsch.) Manuel - ITA, NVF, RJN, TER; 0-1350 m

Zelometeorium patens (Hook.) Manuel - ITA, NVF, TER; 0-1400 m

Zelometeorium patulum (Hedw.) Manuel - ANG, CAB, ITA, MAG, MAN, NIT, NVF, PAR, PET, RJN, $\mathrm{SVJ} ; 0-1400 \mathrm{~m}$

Zelometeorium recurvifolium (Hornsch.) Manuel - ANG, CAB, ITA, NIT, NVF, NVI, MAN, PAR, PET, RJN, TER; 0-1350m

\section{Bruchiaceae (3/9)}

Eobruchia bruchioides (Müll. Hal.) W.R. Buck - ITA; 2000-2430 m; VU 
Pringleella subulata (Müll. Hal.) Broth. - ITA; ca. 2300 m; VU

Trematodon ambiguus (Hedw.) Hornsch. - RJN; 600-800 m; VU

Trematodon brevifolius Broth. - ITA; 1100-2300 m; DD

Trematodon gymnostomus Lindb. - ITA; 500-2000 m

Trematodon heterophyllus Müll. Hal. - ITA; 1000-2000 m; DD

Trematodon longicollis Michx. - ANG, MAN, NIT, NVF, PET, RJN, TER; 0-800 m

Trematodon pauperifolius Müll. Hal. - ITA; 800-2000 m; VU

Trematodon vaginatus Müll. Hal. - RJN; 500-900 m

Bryaceae (5/36)

Anomobryum conicum (Hornsch.) Broth. - ITA, NVF; 800-1200 m

Brachymenium hornschunchianum Mart. - ITA, NVF, PET, RJN, TER; 500-2000 m

Brachymenium morasicum Besch. - ITA; 950-2300 m

Brachymenium radiculosum (Schwägr.) Hampe- ITA, MAG, NVF, PET, RJN, TER;800-2000 m

Brachymenium systylium (Müll. Hal.) A. Jaeger - ANG, NIT; 0-1100 m

Bryum apiculatum Schwägr. - S/L; 0-1100 m

Bryum argenteum Hedw. - ACA, ANG, CAF, ITA, NIT, NVF, PET, RJN, SAQ, TER; 0- $2100 \mathrm{~m}$

Bryum atrovirens Brid. - TER; ca. $1200 \mathrm{~m}$; DD

Bryum brasiliense Hampe - S/L; 0-500 m; EN

Bryum brevicoma Hampe - S/L; s/alt.; DD

Bryum caespiticium L. - RJN, TER; 800-1200 m

Bryum clavatum (Schimp.) Müll. Hal. - S/L; s/alt.; DD

Bryum coronatum Schwägr. - S/L; 0-1100 m

Bryum densifolium Brid. - ANG, CAB, ITA, MAG, MAN, NIT, NVF, RJN, TER; 0-1200 m

Bryum dichotomum Hedw. - S/L; nível do mar; DD

Bryum gracilisetum Hornsch. - ITA, NVF, PET; 0-2100 m

Bryum limbatum Müll. Hal. - CAB, MAN, NIT, NVF, PAR, RJN, TER; 0-800 m

Bryum multiflorum Müll. Hal. - RJN; ca. 1200 m; EN

Bryum oncophorum Hampe - S/L; s/alt.; DD

Bryum pabstianum Müll. Hal. - ANG, NIT, RJN, TER; 0-1250 m

Bryum paradoxum Schwägr. - ANG, MAG, MAN, NIT, NVF, PET, RJN, TER; 0-1200 m

Bryum pseudocapillare Besch. - RJN; nível do mar; VU

Bryum renauldii Röll - ANG, NIT, RJN; nível do mar; VU

Bryum subapiculatum Hampe - NVF, RJN; 0-800 m

Bryum torquatum Mohamed. - S/L; 0-200 m; DD

Rhodobryum aubertii (Schwägr.) Thér. - NVF, RJN; 0-1200 m; DD

Rhodobryum beyrichianum (Hornsch.) Müll. Hal. - MAG, NVF, NVI, PAR, PET, RJN, TER; 0-2200 m

Rhodobryum grandifolium (J. Taylor) Schimp. - ITA, RJN; 700-2250 m

Rhodobryum horizontale Hampe - NVF, RJN, TER; 800-1200 m; DD

Rhodobryum huillense (Welw. \& Duby) Touw - ITA, PET; 0-1400 m

Rhodobryum pseudomarginatum (Geh. \& Hampe) Paris - PAR, SVJ; 50-500 m

Rhodobryum roseolum Müll. Hal. - ITA, NVF, TER; 100-2200 m

Rhodobryum roseum (Hedw.) Limpr. - ITA; 440-2400 m; VU

Rhodobryum subverticillatum Broth. - ITA, NVF, PAR, SVJ; 0-2000 m

Rosulabryum billarderi (Schwägr.) Spence - ANG, CAB, ITA, MAN, NIT, NVF, PAR; 0- 2100 m

Rosulabryum capillare (Hedw.) Spence - ITA, NIT, RJN; 0-1200 m

Calymperaceae (3/26)

Calymperes afzelii Sw. - ANG, CAB, MAN, NIT, PAR; 0-600 m

Calymperes erosum Müll. Hal. - ANG, MAN, NIT; 0-650 m

Calymperes lonchophyllum Schwägr. - ANG, CAB, NIT, NVF, NVI, PAR, PET, RJN; 0-1200 m

Calymperes palisotii Schwägr. - ACA, MAC, MAR, NIT; 0-500 m

Calymperes palisotii subsp. richardii (Müll. Hal.) S. Edwards - ACA, ANG, MAN, RJN, TRR; 0-200 m 
Calymperes smithii E.B. Bartram - PAR, SVJ; 0-900 m; DD

Calymperes tenerum Müll. Hal. - CAF, MAC, PAR, RJN, SVJ; 0-200 m

Octoblepharum albidum Hedw. - ANG, CAB, ITA, MAC, MAG, MAN, NIT, NVF, NVI, PAR, PET, QUI, RES, RJN, ROS, SVJ, TER; 0-1200 m

Octoblepharum cocuiense Mitt. - ANG, MAN, NIT, PAR, RJN, SVJ; 0-1200 m

Octoblepharum pulvinatum (Dozy \& Molk.) Mitt. - ANG, NVI, PAR, RJN; 0-2000 m

Syrrhopodon elongatus var. glaziovii (Hampe) W.D. Reese - S/L; 0-1100 m

Syrrhopodon gardneri (Hook.) Schwägr. - NVF; 0-1200 m

Syrrhopodon gaudichaudii Mont. - ANG, ITA, NIT, NVF, PAR, RJN, TER; 0-1200 m

Syrrhopodon incompletus var. berteroanum Schwägr. - PAR; 0-1000 m

Syrrhopodon incompletus var. incompletus Schwägr. - ANG, MAN, NIT, PAR, RJN, SVJ; 0-800 m

Syrrhopodon lanceolatus (Hampe) W.D. Reese - RJN; 0-800 m

Syrrhopodon ligulatus Mont. - RJN; 0-1000 m

Syrrhopodon lycopodioides (Brid.) Müll. Hal. - ANG, NIT; 600-800 m

Syrrhopodon parasiticus (Brid.) Paris - ACA, CAB, MAN, NIT, NVF, SVJ; 0-1000 m

Syrrhopodon prolifer var. acanthoneuros (Müll. Hal.) Müll. Hal. - NIT, NVF, PET, RJN, TER; 0-2200 m

Syrrhopodon prolifer var. cincinnatus Hampe - RJN; 500-1000 m

Syrrhopodon prolifer var. prolifer Schwägr. - ANG, CAB, MAN, NIT, NVF, PET, RJN, SVJ, TER; 0-1500 m Syrrhopodon prolifer var. scaber (Mitt.) W.D. Reese - ANG, NIT; 0-800 m

Syrrhopodon prolifer var. tenuifolius (Sull.) W.D. Reese - NVF, PET, RJN, TER; 0-1600 m

Syrrhopodon rigidus Hook. \& Grev. - ANG, MAN, NIT; 0-500 m

Syrrhopodon tortilis Hampe - ITA, RJN; 800-1200 m

Catagoniaceae (1/2)

Catagonium brevicaudatum Müll. Hal. - ITA; 1500-2750 m

Catagonium emarginatum S.H. Lin - ITA; 2200-2550 m

Cryphaeaceae (2/3)

Cryphaea malmei Broth. - ITA; 0-1200 m

Cryphaea raddiana (Brid.) Hampe - MAG; ca. 800 m; DD

Schoenobryum concavifolium (Griff.) Gangulee - ITA, NVF, PET, TER; 0-2000 m

Daltoniaceae (4/7)

Calyptrochaeta albescens (Hampe) W.R. Buck - S/L; s/alt; VU

Calyptrochaeta setigera (Mitt.) W.R. Buck - NVF, PET, TER; 0-2200 m

Daltonia aristata Geh. \& Hampe - ITA; 0-2500 m

Daltonia brasiliensis Mitt. - ITA; 800-2100 m

Distichophyllum gracile Aongstr. - S/L; ca. 1100 m; DD

Distichophyllum minutum Müll. Hal. - NVF, RJN; 0-1000 m; DD

Leskeodon aristatus (Geh. \& Hampe) Broth. - MAG, PET, RJN; 900-1700 m

Dicranaceae (13/64)

Atractylocarpus brasiliensis (Müll. Hal.) R.S. Williams - ITA, PET; 1900-2300 m; VU

Atractylocarpus longisetus (Hook.) E.B. Bartram - ITA, TER; 1200-2700 m; VU

Bryohumbertia filifolia (Hornsch.) J.-P. Frahm var. filifolia - ANG, CAB, ITA, MAG MAN, NIT, NVF, NVI, PET, RJN, TER; 0-2000 m

Bryohumbertia filifolia var. humilis (Mont.) J.-P. Frahm - ITA, PET, TER; 800-2500 m

Campylopus aemulans (Hampe) A. Jaeger - ITA, RJN, TER; 800-2800 m

Campylopus albidovirens Herzog - NVI; 1500 m; VU

Campylopus angustiretis (Austin) Lesq. \& James - ITA, RJN; 800-2250 m

Campylopus arctocarpus (Hornsch.) Mitt. var. arctocarpus - ANG, ITA, NVF, NVI PAR, PET, RJN, TER; 0-2000 m

Campylopus arctocarpus var. caldensis (Aongstr.) J.-P. Frahm - ITA, NIT, NVF, PAR, PET, TER; 1000$2000 \mathrm{~m}$ 
Campylopus cryptopodioides Broth. - ACA, ANG, ARA, MAC, MAG, MAR, NIT, NVF, PET, ROS, SAQ, SVJ, TER; 0-1500m

Campylopus cuspidatus (Hornsch.) Mitt. var. cuspidatus - ITA; 800-1600 m

Campylopus cuspidatus var. dicnemoides (Müll. Hal.) J.-P. Frahm - ITA; 1500-2000 m; VU

Campylopus densicoma (Müll. Hal.) Paris - ITA; 1500-2600 m; VU

Campylopus dichrostis (Müll. Hal.) Paris - NVF, PAR, PET, RJN, TER; 0-800 m

Campylopus flexuosus (Hedw.) Brid. - RJN; ca. 800 m; DD

Campylopus fragilis subsp. fragiliformis (J.-P. Frahm) J.-P. Frahm - ITA, TER; 1500-2500 m

Campylopus fuscocroceus (Hampe) A. Jaeger - NVF; ca. 2000 m; VU

Campylopus gardneri (Müll. Hal.) Mitt. - NVF; 250-1900 m

Campylopus gemmatus (Müll. Hal.) Paris - ITA, NVF, PAR, RJN, TER; 800-2400 m

Campylopus griseus (Hornsch.) A. Jaeger - ITA, NVF, RJN; 200-2000 m

Campylopus heterostachys (Hampe) A. Jaeger - ANG, ITA, MAN, NIT, NVF; 200-1500 m

Campylopus introflexus (Hedw.) Brid. - NVF; 0-1200 m

Campylopus jamesonii (Hook.) A. Jaeger - ITA; 900-2700 m; VU

Campylopus julaceus A. Jaeger - ITA, NVF; 300-1500 m

Campylopus julicaulis Broth. - ITA, NVF, RJN; 0-2200 m

Campylopus lamellinervis (Müll. Hal.) Mitt. - ITA, NVF, NVI, RJN, TER; 0-2500 m

Campylopus occultus Mitt. - ITA, MAC, MAR, NVF, PAR, PET, QUI, ROS, RJN, SJB, TER; 0-2500 m

Campylopus pilifer Brid. - ANG, ITA, NIT, RJN, TER; 0-2500 m

Campylopus reflexisetus (Müll. Hal.) Broth. - ITA, NVF, NVI; 2500-2700 m

Campylopus richardii Brid. - ITA, NVF; 600-2700 m

Campylopus savannarum (Müll. Hal.) Mitt. - ACA, ANG, MAN, NIT, NVF; 0-1500 m

Campylopus subcuspidatus (Hampe) A. Jaeger - RJN; 0-800 m

Campylopus surinamensis Müll. Hal. - ITA, RJN; 0-1500 m

Campylopus thwaitesii (Mitt.) A. Jaeger - ANG, ITA, NVF, PAR, RJN, TER; 800-2500 m

Campylopus trachyblepharon (Müll. Hal.) Mitt. - ANG, MAC, MAG, MAN, MAR, NIT, PAR, PET, RJN, ROS; 0-800 m

Campylopus uleanus (Müll. Hal.) Broth. - NIT, PET, TER; 0-1900 m

Campylopus widgrenii (Müll. Hal.) Mitt. - RJN; ca. $800 \mathrm{~m}$

Dicranella exigua (Schwägr.) Mitt. - MAG, NVF, RJN; 0-800 m

Dicranella guilleminiana (Mont.) Mitt. - ITA, PET, RJN, TER; 800-1400 m

Dicranella gymna (Müll. Hal.) Broth. - ITA; 1700 m; DD

Dicranella hilariana (Mont.) Mitt. - ANG, CAB, NIT, NVF, PAR, PET, TER; 0-2000 m

Dicranella itatiaiae (Müll. Hal.) Broth. - ITA; 2000 m; DD

Dicranella longirostris (Schwägr.) Mitt. - MAG, RJN; 600-1200 m; DD

Dicranella martiana (Hampe) Hampe - ITA, RES, RJN; 700-1100 m

Dicranella subsulcata (Hampe) Hampe - S/L; s/alt; DD

Dicranella ulei (Müll. Hal.) Broth. - ITA; 1100-1500 m; DD

Dicranodontium pulchroalare subsp. brasiliense (Herzog) J.-P. Frahm - TER; ca. 1200 m; DD

Dicranoloma brasiliense Herzog - PET, TER; 1600-2200 m; DD

Dicranoloma subenerve Herzog - PET, TER; ca.1200 m; DD

Dicranum frigidum Müll. Hal. - ITA, NVF; 1500-2200 m

Holomitrium antennatum Mitt. - PET, TER; 0-2200 m

Holomitrium arboreum Mitt. - ANG, NIT, NVF, NVI; 0-1200 m

Holomitrium crispulum Mart. - ANG, ITA, NIT, NVF, PET, RJN, TER; 0-2200 m

Holomitrium glaziovii Geh. \& Hampe - S/L; ca. 900 m

Holomitrium olfersianum Hornsch. - ANG, ITA, MAN, NIT, NVF, RJN, TER; 0-2500 m

Leucoloma biplicatum (Hampe) A. Jaeger - ITA, NVF, RJN; 0-1600 m

Leucoloma cruegerianum (Müll. Hal.) A. Jaeger - ANG, NIT, NVF; 500-1100 m

Leucoloma itatiaiense Broth. - ITA; 2200 m; VU

Leucoloma serrulatum Brid. - ANG, CAB, MAN, NIT, NVF, NVI, PAR, PET; 0-1400 m 
Leucoloma triforme (Mitt.) A. Jaeger - RJN, TER; 0-1300 m; VU

Microcampylopus curvisetus (Hampe) Giese \& J.-P. Frahm.- ITA; 0-2000 m

Oreoweisia brasiliensis Hampe - ITA; 2300 m; VU

Paraleucobryum longifolium subsp. brasiliense (Broth.) P. Mueller \& J.-P. Frahm - ITA; 2500-2800 m; VU

Pilopogon guadalupensis (Brid.) J.-P. Frahm - ITA, NVF, PAR, PET, RJN, TER; 600-2000 m

\section{Diphysciaceae (1/1)}

Diphyscium peruvianum Spruce - ITA, TER; 0-1300 m

\section{Ditrichaceae (5/9)}

Ceratodon pupureus subsp. stenocarpus (B.S.G.) Dix. - ITA; 1100-2890 m

Chrysoblastella chilensis (Mont.) Reimers - ITA, PET; 2100 m; VU

Cladastomum robustum Broth. - ITA; 2000-2500 m; VU

Cladastomum ulei Müll. Hal - ITA; 1400-2580 m

Crumuscus vitalis W.R. Buck \& Snider - ITA; 2100-2700 m; EN

Ditrichum itatiaiae (Müll. Hal.) Paris var. itatiaiae - ITA; 1400-1700 m; VU

Ditrichum itatiaiae var. brevipes (Müll. Hal.) Paris - ITA; 2000-2500 m; VU

Ditrichum liliputanum (Müll. Hal.) Paris - ITA; 950-2000 m; VU

Ditrichum ulei (Müll. Hal.) Paris - ITA; 0-2500 m

Entodontaceae (2/9)

Entodon beyrichii (Schwägr.) Müll. Hal. - CAB, MAN, NIT, NVF, RJN, TER; 0-800 m

Entodon hampeanus Müll. Hal. - NVF; ca. $620 \mathrm{~m}$; VU

Entodon jamesonii (J. Taylor) Mitt. - ITA, RJN, TER; 1200-2500 m

Entodon lindbergii Hampe - NVF; 500-1100 m

Entodon mosenii Broth. - NVF; ca. $1100 \mathrm{~m}$; VU

Entodon splendidulus Hampe - ITA, TER; 600-1200 m

Entodon virens (Hook. \& Wilson) Mitt. - TER; ca. 1200 m; VU

Erythrodontium longisetum (Hook.) Paris - MAN, NIT, NVF, PET, RJN, TER; 0-800 m

Erythrodontium squarrosum (Hampe) Paris - PET; 0-1350 m

Ephemeraceae (2/2)

Ephemerum pachyneuron Müll. Hal. - ITA; 1500-2800 m

Micromitrium austinii Sull. - ITA, RJN; nível do mar; VU

Erpodiaceae (1/2)

Erpodium coronatum (Hook. \& Wilson) Mitt. - S/L; 0-1200 m

Erpodium glaziovii Hampe - BAP, ITA, MAN, NIT, RJN; 0-800 m

Fabroniaceae (2/3)

Dimerodontium pellucidum (Schwägr.) Mitt. - S/L; ?-700 m; DD

Fabronia ciliaris var. polycarpa (Hook.) W.R. Buck - CAB, RJN; 0-900 m

Fabronia ciliaris var. wrightii (Sull.) W.R. Buck - ANG, MAN, NIT; nível do mar

Fissidentaceae (1/28)

Fissidens acacioides Schrader - S/L; 0-1100 m

Fissidens angustelimbatus Mitt. - ITA, NVF; 0-2000 m

Fissidens angustifolius Sull. - NIT; 0-1000 m

Fissidens asplenioides Hedw. - ANG, ITA, MAN, NIT, NVF, PAR, PET, RJN, TER; 0-2200 m

Fissidens elegans Brid. - ANG, NIT; 0-1100 m

Fissidens flaccidus (Mitt.) Mitt. - ANG, RJN, SVJ; 0-1100 m

Fissidens gardneri Mitt. - RJN; 0-600 m

Fissidens guianensis Mont. - ACA, ANG, MAC, NIT, RJN; 0-500 m

Fissidens hornschuchii Mont. - ITA, MAG, NIT, NVF, PAR, PET, RJN; 0-2000 m

Fissidens inaequalis Mitt. - RJN; nível do mar

Fissidens intramarginatus (Hampe) A. Jaeger - ANG, MAN, NIT, NVF, RJN, TER; 0-1200 m 
Fissidens lagenarius Mitt. - ANG, MAN, NIT; 0-1350 m

Fissidens oediloma Müll. Hal. - PET, TER; 800-2200 m

Fissidens palmatus Hedw. - RJN; 0-800 m

Fissidens pellucidus var. asterodontius (Müll. Hal.) Pursell - ITA; 0-2000 m

Fissidens pellucidus var. papilliferus (Broth.) Pursell - RJN; nível do mar

Fissidens pellucidus Hornsch. var. pellucidus - ANG, MAG, NIT, PET, RJN, TER; 0-2200 m

Fissidens prionodes Mont. - NVF; 0-1500 m

Fissidens radicans Mont. - PAR, RJN; 0-200 m

Fissidens reticulosus (Müll. Hal.) Mitt. - RJN; 0-1100 m

Fissidens rigidulus Hook. \& Wilson - CAB, ITA; 0-1200 m

Fissidens scariosus Mitt. - ANG, CAB, NIT, NVF; 0-1350 m

Fissidens serratus Müll. Hal. - RJN; 0-900 m

Fissidens submarginatus Bruch - MAC, MAR, RJN; 0-500 m

Fissidens wallisii Müll. Hal. - ITA; 1500-2880 m; VU

Fissidens weirii var. hemicraspedophyllus (Cardot) Pursell - NVF; ca. 1000 m; VU

Fissidens weirii Mitt. var. weirii - RJN; 0-800 m

Fissidens zollingeri Mont. - ACA, ANG, CAB, MAN, MAC, NIT, PAR, RJN, SAQ, SVJ; 0- 800 m

\section{Fontinalaceae (1/1)}

Fontinalis squamosa var. curta Arnott - S/L; s/alt; DD

\section{Funariaceae (3/6)}

Entosthodon bonplandii (Hook.) Mitt. - ITA, RJN, TER; 0-2300 m

Funaria beyrichii Hampe - S/L; nível do mar; DD

Funaria hygrometrica var. calvescens (Schwägr.) Kindb. - ITA, NVF, PET, RJN, TER; 0- $2890 \mathrm{~m}$

Funaria hygrometrica Hedw. var. hygrometrica - NVF, TER; 0-1200 m

Funaria ramulosa (Hampe) Paris - S/L; s/alt; DD

Physcomitrium acutifolium Broth. - RJN; 0-200 m; VU

\section{Grimmiaceae (2/4)}

Grimmia elongata Kauf. - ITA; ca. 2100 m; VU

Grimmia longirostris Hook. - ITA; 1200-2770

Racomitrium crispulum (J. Taylor) A. Jaeger - ITA; 1200-2500 m

Racomitrium tortipilum (Müll. Hal.) Broth. - ITA; 2000-2500 m; VU

\section{Hedwigiaceae (1/2)}

Hedwigidium glyphocarpum (Hampe) A. Jaeger - ITA; 200-2500 m

Hedwigidium integrifolium (P. Beauv.) Dixon - NVF; 0-2890 m

Helicophyllaceae (1/1)

Helicophyllum torquatum (Hook.) Brid. - ANG, CAB, CAM, CAR, ITA, MAC, MAN, MAR, MIR, NIT, NVI, PAR, RJN, SAP, STA, SVJ; 0-1200 m

\section{Hookeriaceae (2/3)}

Eriopus flexicaulis (Hampe) Paris - RJN, TER; ca. 1200 m; DD

Eriopus lorifolius (Hampe) Paris - S/L; s/alt; DD

Hookeria acutifolia Hook. \& Grev. - NVF, TER; 0-1500 m

\section{Hydropogonaceae (1/1)}

Hydropogon fontinaloides (Hook.) Brid. - RJN; nível do mar

\section{Hypnaceae (9/37)}

Chryso-hypnum diminutivum (Hampe) W.R. Buck - ITA, NVF, RJN; 0-1200 m

Chryso-hypnum elegantulum (Hook.) Hampe - ANG, CAB, MAN, NIT, NVF, NVI, PAR, RJN, TER; 0$1200 \mathrm{~m}$

Ctenidium malacodes Mitt. - ITA; 1200-2750 m 
Ectropothecium campaniforme (Müll. Hal.) Paris - TER; ca. 1200 m; DD

Ectropothecium cupressoides (Müll. Hal.) Mitt. - RJN; ca. 600 m; DD

Ectropothecium cylindricum Mitt. - MAG; ca. 800 m; DD

Ectropothecium hypnoides (Hornsch.) A. Jaeger - RJN; nível do mar; DD

Ectropothecium leptochaeton (Schwägr.) W.R. Buck - NVF, PAR, RJN, TER; 0-1350 m

Ectropothecium urceolatum (Hornsch.) Mitt. - S/L; s/alt; DD

Hypnum amabile (Mitt.) Hampe - ITA, TER; 0-2750 m; VU

Isopterygium affusum Mitt. - ITA; 0-1950 m

Isopterygium subbrevisetum (Hampe) Broth. - NVF, PET, SVJ; 0-1200 m

Isopterygium tenerifolium Mitt. - ANG, CAB, ITA, MAN, MAR, NIT, NVF, PAR, RJN, SVJ; 0-2000 m

Isopterygium tenerum (Sw.) Mitt. - ANG, CAB, ITA, MAC, MAG, MAN, MAR, NIT, NVF, PAR, PET, RJN, SVJ, SAQ, TER; 0-1350 m

Mittenothamnium heterostachys (Hampe) Cardot - NVF, PET, TER; 800-1200 m

Mittenothamnium langsdorffii (Hook.) Cardot - ITA, NVF, PET, TER; 0-2750 m

Mittenothamnium macrodontium (Hornsch.) Cardot - NVF, RJN; 0-1200 m

Mittenothamnium pachythecium (Hampe) Cardot - S/L; s/alt; DD

Mittenothamnium reduncum (Mitt.) Ochyra - ITA, TER; 430-2300 m

Mittenothamnium reptans (Hedw.) Cardot - NVF, PAR, TER; 0-1350 m

Mittenothamnium simorrhynchum (Hampe) Cardot - ITA, NVF, PET, RJN; 0-1200 m

Mittenothamnium subdiminutivum (Geh. \& Hampe) Cardot - NVF, PAR; 700-1000 m

Mittenothamnium submacrodontium (Geh. \& Hampe) Cardot - NVF; 0-950 m

Mittenothamnium tamarisciforme (Hampe) Cardot - S/L; s/alt; DD

Mittenothamnium versipoma (Hampe) Cardot - ITA, NVF; 0-1000 m

Phyllodon truncatulus (Müll. Hal.) W.R. Buck - RJN; 0-600 m; DD

Taxiphyllum taxirameum (Mitt.) M. Fleisch. - CAB; 500-800 m; VU

Vesicularia aquatilis Müll. Hal. - S/L; s/alt; DD

Vesicularia glaucopinnata Müll. Hal. - RJN; 0-1000 m

Vesicularia glazioviana Müll. Hal. - S/L; s/alt; DD

Vesicularia pelvifolia Müll. Hal. - ITA, MAG; s/alt; DD

Vesicularia sigmatellopsis Müll. Hal. - S/L; s/alt; DD

Vesicularia tophacea Müll. Hal. - S/L; s/alt; DD

Vesicularia trullifolia Müll. Hal. - MAG; 500-1100 m; DD

Vesicularia vesicularis var. portoricensis (Brid.) W.R. Buck - S/L; 0-? m

Vesicularia vesicularis var. rutilans (Brid.) W.R. Buck - ANG, MAN; nível do mar

Vesicularia vesicularis (Schwägr.) Broth. var. vesicularis - ANG, CAB, MAN, NIT, NVI, PAR, RJN; $0-800 \mathrm{~m}$

\section{Hypopterigyaceae (2/5)}

Hypopterygium flavescens Hampe - MAG, NVF, PAR, RJN, TER; 0-1100 m

Hypopterygium laricinum (Hook.) Brid. - MAG, NVF, TER; 0-1200 m

Hypopterygium monoicum Hampe - ITA, MAG, NVF, PAR, PET, RJN, TER; 0-1200 m

Hypopterygium tamarisci (Sw.) Brid. - ANG, ITA, MAN, NIT; 0-1350 m

Lopidium concinnum (Hook.) Wilson - ANG, ITA, NIT, NVF, RJN, TER; 0-1200 m

\section{Lembophyllaceae (2/11)}

Orthostichella microcarpa Müll. Hal. - NVF, PAR, RJN; 0-1100 m

Orthostichella mucronatula Müll. Hal. - NVF; 0-1100 m

Orthostichella versicolor (Müll. Hal.) B.H. Allen \& W.R. Buck - ANG, ITA, MAG, MAN, NIT, NVF, NVI, PAR, PET, RJN, TER; 0-1800 m

Pilotrichella flexilis (Hedw.) Aongstr. - ANG, ITA, NIT, NVF, NVI, TER; 0-2300 m

Pilotrichella flexilis fo. nudiramulosa (Müll. Hal.) Allen \& Magill - NVF; 0-1400 m

Pilotrichella pachygastrella Müll. Hal. - PET, RJN; 0-1100 m

Pilotrichella pallidicaulis Müll. Hal. - ITA, NVF; 700-2000 m 
Pilotrichella rigida (Müll. Hal.) Besch. - NVI; 600-1500 m

Pilotrichella squarrulosa var. squarrulosa Müll. Hal. - ITA, NVF, PAR, PET, RJN, TER; 600-1800 m

Pilotrichella subpachygastrella Broth. - NVF; 0-1000 m

Pilotrichella welwitschii (Duby) Gepp - TER; ca. 1200 m; DD

Leskeaceae (1/1)

Haplocladium microphyllum (Hedw.) Broth. - ANG, MAN, NIT, RJN, TER; 0-800 m

\section{Leucobryaceae (2/10)}

Leucobryum albicans (Schwägr.) Lindb. - ANG, CAB, ITA, NIT, NVF, PET, RES, RJN, TER; 0-2200 m

Leucobryum albidum (Brid.) Lindb. - ITA, PAR, RES; 0-1500 m

Leucobryum clavatum var. brevifolium Broth. - RJN; 0-1050 m

Leucobryum clavatum Hampevar. clavatum - ANG, CAB, ITA, NIT, NVF, PET, RJN, TER; 0-2200 m

Leucobryum crispum Müll. Hal. - ANG, ITA, MAN, NIT, NVF, RJN, TER; 0-1800 m

Leucobryum giganteum Müll. Hal. - ITA, NVF, NVI, RJN, TER; 0-1850 m

Leucobryum juniperoideum (Brid.) Müll. Hal. - TER; 0-800 m

Leucobryum martianum (Hornsch.) Hampe - ANG, MAN, NIT, NVF, NVI, PAR, RJN, SVJ; 0-1000 m

Leucobryum sordidum Aongstr. - ITA, MAG, NVF, PAR, PET, RJN, TER; 500-1400 m

Ochrobryum gardneri (Müll. Hal.) Lindb. - S/L; 0-1200 m

Leucodontaceae (3/3)

Felipponea montevidensis (Müll. Hal.) Broth. - TER; 500-1200 m

Pterogonidium pulchellum (Hook.) Müll. Hal. - CAB, NVF, RJN, SVJ; 0-800 m

Pterogonium beyrichianum Hampe - NVF; ca. 800 m; DD

Leucomiaceae (2/3)

Leucomium steerei Allen \& Veling - TER; 1170 m; VU

Leucomium strumosum (Hornsch.) Mitt.- ANG, MAG, MAN, NIT, PAR, RJN, SVJ, TER; 0-1350 m

Philophyllum tenuifolium (Mitt.) Broth. - ITA, NVF; 0-2200 m

Meteoriaceae (7/12)

Cryptopapillaria penicillata (Dozy \& Molk.) M. Menzel - NVF; 0-1100 m

Floribundaria flaccida (Mitt.) Broth. - ANG, CAB, MAN, NIT, NVF, TER; 0-1200 m

Meteoriopsis aureonitens (Hornsch.) Broth. - ITA, NVF, PET; 0-2000 m

Meteorium deppei (Müll. Hal.) Mitt. - ANG, CAB, ITA, MAN, NIT, NVF, PAR, RJN, TER; 0-2000 m

Meteorium nigrescens (Sw.) Dozy \& Molk. - ANG, CAB, ITA, MAN, NIT, NVF, NVI, PAR, PET, RJN, TER; 0-2000 m

Papillaria capillicuspis Müll. Hal. - RJN; 0-900 m

Papillaria crenifolia Müll. Hal. - NVF; 0-800 m; DD

Papillaria mosenii Broth. - PET, RJN; 0-1200 m

Papillaria subintegra (Lindb.) Paris - S/L; ?-900 m; DD

Papillaria tijucae Müll. Hal. - RJN; ca. 1200 m; DD

Toloxis imponderosa (J. Taylor) W.R. Buck - ITA; 500-1200 m

Trachypus bicolor var. hispidus (Müll. Hal.) Cardot - ITA; 0-2300 m

Mielichhoferiaceae (2/5)

Mielichhoferia grammocarpa Müll. Hal. - ITA; ca. 2500 m; DD

Mielichhoferia striidens Müll. Hal. - ITA; 2000 m; DD

Mielichhoferia ulei Müll. Hal. - ITA; 0-1200 m

Schizymenium brevicaule (Hornsch.) A.J. Shaw \& S.P.Churchill - S/L ; ?-1200 m

Schizymenium linearicaule (Müll. Hal.) A.J. Shaw - ITA; ca. 1200 m; DD

Mniaceae (3/10)

Epipterygium puiggarii (Geh. \& Hampe) Broth. - MAG; 800-1000 m; DD

Plagiomnium rhynchophorum (Hook.) T.J. Kop. - ITA, MAG, MAN, NIT, NVF, PAR, PET, RES, RJN, TER; 0-2750 m 
Plagiomnium rostratum (Schrad.) T.J. Kop. - NVI, TER; 0-1200 m

Pohlia camptotrachela (Renauld \& Cardot) Broth. - ITA; 1200-1550 m

Pohlia crassicostata (Müll. Hal.) Broth. - ITA; 1200-2000 m; DD

Pohlia cf. elongata Hedw. - NIT; 0-2500 m

Pohlia grammocarpa (Müll. Hal.) Broth. - ITA; 1000-2500 m; DD

Pohlia leptopoda (Hampe) Broth. - S/L; s/alt; DD

Pohlia papillosa (A. Jaeger) Broth. - TER; ca. 1900 m; VU

Pohlia tenuifolia (A. Jaeger) Broth. - PET; RES; 800-1400 m

Myriniaceae (2/2)

Helicodontium capillare (Hedw.) A. Jaeger - ANG, CAB, MAG, MAN, NIT, NVF, PET, RJN, SVJ, TER; $0-1200 \mathrm{~m}$

Myrinia brasiliensis (Hampe) Schimp. - S/L; 0-900 m

\section{Neckeraceae (9/17)}

Homalia glabella (Hedwig) Schimp. - PET; 0-1000 m

Homaliodendron piniforme (Brid.) Enroth - PAR; nível do mar; VU

Isodrepanium lentulum (Wilson) E. Britton - PAR; nível do mar

Neckera caldensis Lindb. - NVF, TER; 450-1630 m

Neckera scabridens Müll. Hal. - ITA, NVF; 500-2000 m

Neckeropsis disticha (Hedw.) Kindb. - ANG, CAB, ITA, MAN, NIT, PAR, RJN, SVJ; 0-1100 m

Neckeropsis undulata (Hedw.) Reichardt. - ANG, CAB, ITA, MAG, MAN, NIT, NVF, NVI, PAR, RJN, SVJ, TER; 0-1200 m

Neckeropsis villae-ricae (Besch.) Broth. - ANG, MAN, NIT, RJN; 0-1000 m

Porothamnium leucocaulon (Müll. Hal.) M. Fleisch. - ITA, NVF; 0-2750 m

Porothamnium obliquifolium (Hornsch.) M. Fleisch. - S/L; s/alt

Porotrichodendron glaziovii (Paris) Wijk \& Margad. - S/L; s/alt; DD

Porotrichum korthalsianum (Dozy \& Molk.) Mitt. - ITA, MAG, NVF, PAR, PET; 200-2000 m

Porotrichum lancifrons (Hampe) Mitt. - ITA, TER; 0-2500 m

Porotrichum longirostre (Hook.) Mitt. - ITA, NVF, NVI, PAR, TER; 800-2700 m

Porotrichum mutabile Hampe - ANG, ITA, MAN, NIT; 0-1700 m

Porotrichum substriatum (Hampe) Mitt.- ANG, CAB, ITA, MAN, NIT, NVF, PAR, RJN; 0-2000 m

Thamnobryum fasciculatum (Hedw.) I. Sastre - PET, TER; 0-1250 m

Orthodontiaceae (1/1)

Orthodontium pellucens (Hook.) B.S.G. - ITA; 500-2000 m

Orthotrichaceae $(5 / 51)$

Groutiella apiculata (Hook.) H.A. Crum \& Steere - CAF, MAG, NIT, PAR, TER; 0-1200 m

Groutiella tomentosa (Hornsch.) Wijk \& Margad. - ANG, CAB, NIT, PAR; 0-500 m

Groutiella tumidula (Mitt.) Vitt - ANG, NIT; nível do mar

Macrocoma frigida (Müll. Hal.) Vitt - ANG, CAB, NIT; 0-910 m

Macrocoma orthotrichoides (Raddi) Wijk \& Margad.- ITA, MAG, NVF, PET, RJN, TER; 0-2500 m

Macrocoma tenue subsp. sullivantii (Müll. Hal.) Vitt. - ITA, RJN; 0-2500 m

Macromitrium argutum Hampe - ANG, BAM, ITA, MAN, NIT; 380-1400 m

Macromitrium catharinense Paris - ITA, PET, TER; 0-2200 m

Macromitrium cirrosum (Hedw.) Brid. - ITA, MAG, NVF, RJN, TER; 0-1150 m

Macromitrium doeringianum Hampe - PET; ca. 800 m; DD

Macromitrium eriomitrium Müll. Hal. - ITA; 1200-2500 m; DD

Macromitrium filicaule Müll. Hal. - S/L; s/alt; DD

Macromitrium glaziovii Hampe - S/L; s/alt; DD

Macromitrium guatemaliense Müll. Hal. - ITA; 1100-1950 m

Macromitrium hornschuchii Müll. Hal. - NVF, RJN, SVJ; 0-1200 m

Macromitrium longifolium (Hook.) Brid. - S/L; s/alt 
Macromitrium microstomum (Hook. \& Grev.) Schwägr. - ANG, NIT; 600-1170 m

Macromitrium nitidum Hook. \& Wilson - ITA, RJN; 0-1200 m

Macromitrium pellucidum Mitt. - ANG, MAN, NIT; 0-1100 m

Macromitrium pseudofimbriatum Hampe - S/L; s/alt; DD

Macromitrium punctatum (Hook. \& Grev.) Brid. - ANG, ITA, MAN, NIT, RJN; 0-2100 m

Macromitrium richardii Schwägr. - ANG, CAB, MAC, MAG, MAN, NIT, NVF, PAR, PET, RJN, TER; $0-1200 \mathrm{~m}$

Macromitrium stellulatum (Hornsch.) Brid. - RJN; 0-1100 m

Macromitrium strictifolium Müll. Hal. - TER; s/alt; DD

Macromitrium subapiculatum Broth. - MAG; 0-1600 m; DD

Macromitrium substrictifolium Müll. Hal. - RJN; ca. 1200 m; DD

Macromitrium swainsonii (Hook.) Brid. - RJN; 500-1500 m

Macromitrium undatum Müll. Hal. - ITA; ca. 1100 m; DD

Macromitrium viticulosum (Raddi) Brid. - RJN; 800-1200 m; DD

Schlotheimia capillaris Hampe - S/L; s/alt; DD

Schlotheimia crumii B.C. Tan - NVF; 1300-1800 m

Schlotheimia elata Mitt. - PET, RJN, TER; 0-1200 m; VU

Schlotheimia fasciculata Mitt. - MAG; 800-1200 m

Schlotheimia fuscoviridis Hornsch. - PET, RJN, TER; 0-1900 m

Schlotheimia glaziovii Hampe - NVF, RJN; 0-1400 m

Schlotheimia grammocarpa Müll. Hal. - ITA; 1000-1200 m; VU

Schlotheimia horridula Müll. Hal. - PET; 800-1200 m; DD

Schlotheimia jamesonii (Arnott) Brid. - ANG, CAB, NIT, RJN; 0-1200 m

Schlotheimia muelleri Hampe - RJN; 450-1200 m; DD

Schlotheimia pseudoaffinis Müll. Hal. - ITA; 0-2500 m; DD

Schlotheimia recurvifolia Hornsch. - TER; 0-1100 m

Schlotheimia rugifolia (Hook.) Schwägr. - ANG, CAB, ITA, MAG, MAN, MAR, NIT, NVF, NVI, PAR, PET, RJN, TER; 0-2700 m

Schlotheimia serricalyx Müll. Hal. - ITA, PET, TER; 0-2000 m

Schlotheimia sublaxa Hampe - MAG; ca. 800 m; DD

Schlotheimia subsinuata Geh. \& Hampe - S/L; 0-800 m; DD

Schlotheimia tecta Hook f. \& Wills. - ITA, NVF, NVI, PET, RJN, TER; 700-2750 m

Schlotheimia torquata (Hedw.) Brid. - ITA, MAG, NVF, PET, RJN, SVJ, TER; 0-1550 m

Schlotheimia trichomitria Schwägr. - ITA; 500-1600 m

Zygodon reinwardtii (Hornsch.) A. Braun - ITA; 900-2750 m

Zygodon schenckei Broth. - TER; ca. 1200 m; DD

Phyllogoniaceae (1/2)

Phyllogonium fulgens (Hedw.) Brid. - MAG, RJN; 550-1200 m

Phyllogonium viride Brid. - ANG, CAB, ITA, MAN, NIT, NVF, NVI, PAR, PET, RES, RJN, TER; 0-2300 m

Pilotrichaceae (11/51)

Brymela fluminensis (Hampe) W.R. Buck - ITA; 800-2500 m

Callicostella apophysata (Hampe) A. Jaeger - S/L; ca. 400 m; DD

Callicostella depressa (Hedw.) A. Jaeger - S/L; 0-?m

Callicostella martiana (Hornsch.) A. Jaeger - PAR; 0-1000 m

Callicostella merkelii (Hornsch.) A. Jaeger - ANG, CAB, MAN, NIT, PAR, RJN; 0-800 m

Callicostella microcarpa Aongstr. - NVF, RJN; 0-800 m

Callicostella pallida (Hornsch.) Aongstr. - ANG, CAB, ITA, MAN, NIT, NVF, PAR, RJN, TER; 0-1300 m

Callicostella paulensis Broth. - NVF, RJN; 0-800 m

Callicostella rufescens (Mitt.) A. Jaeger - PAR; 0-200 m; DD

Crossomitrium patrisiae (Brid.) Müll. Hal. - ANG, CAB, MAN, NIT, PAR, RJN, SVJ, TER; 0-800 m

Cyclodictyon albicans (Hedw.) O. Kuntze - ITA, NVF; 800-1600 m 
Cyclodictyon cuspidatum O. Kuntze - S/L; s/alt; DD

Cyclodictyon laxifolium Herzog - PET; ca. 2200 m; EN

Cyclodictyon leucomitrium (Müll. Hal.) Broth. - S/L; 0-1000 m

Cyclodictyon limbatum (Hampe) O. Kuntze - ITA; 0-1200 m

Cyclodictyon olfersianum (Hornsch.) O. Kuntze - NVF, PET, RJN; 0-1100 m

Cyclodictyon rivale (Müll. Hal.) Broth. - RJN; 300-900 m

Cyclodictyon varians (Sull.) O. Kuntze - ANG, CAB, MAN, NIT; 0-340 m

Hookeriopsis beyrichiana (Hampe) Broth. - ITA, PET, RJN, TER; 0-1200 m

Hookeriopsis brachypelma (Müll. Hal.) Broth. - RJN; nível do mar; DD

Hookeriopsis hydrophylla (Müll. Hal.) Broth. - RJN; 0-1000 m

Hookeriopsis puiggarii (Geh. \& Hampe) Broth. - NVF; 800-1100 m; VU

Hookeriopsis rubens (Müll. Hal.) Broth. - NVF, TER; 0-1200 m

Hypnella pallescens (Hook.) A. Jaeger - RJN; 0-800 m

Hypnella pilifera (Hook. \& Wilson) A. Jaeger - NVF, TER; 0-2000 m

Hypnella punctata Broth. - TER; s/alt; DD

Lepidopilidium aureo-purpureum (Geh. \& Hampe) Broth. - RJN; 0-1000 m; EN

Lepidopilidium brevisetum (Hampe) Broth. - ITA, RJN, SVJ; 0-1200 m

Lepidopilidium caudicaule (Müll. Hal.) Broth. - TER; 1200-1700 m; VU

Lepidopilidium entodontella (Broth.) Broth. - MAC, NVF; 0-1600 m; DD

Lepidopilidium laevisetum (Hampe) Broth. - NVF; 0-1500 m

Lepidopilidium nitens (Hornsch.) Broth. - NVF; 0-1000 m

Lepidopilidium plebejum (Müll. Hal.) Sehnem - NVF, RJN; 0-1400 m

Lepidopilidium wainioi (Broth.) Broth. - MAG; 800-1200 m; DD

Lepidopilum affine Müll. Hal. - ITA, TER; 0-2000 m

Lepidopilum flavescens Geh. \& Hampe - TER; 250-1700 m

Lepidopilum ovalifolium (Duby) Broth. - NVF; 0-800 m

Lepidopilum pringlei Cardot - NVF; 700-1300 m

Lepidopilum scabrisetum (Schwägr.) Steere - ANG, CAB, MAG, MAN, NIT, NVF, PAR; 0-1700 m

Lepidopilum subsubulatum Geh. \& Hampe - ITA, NVF; 0-2000 m

Pilotrichum evanescens (Müll. Hal.) Müll. Hal. - MAG, MAN, NIT; 0-1200 m

Thamniopsis incurva (Hornsch.) W.R. Buck - ANG, CAB, ITA, MAN, NIT, NVF, NVI, PAR, PET, RJN, SVJ, TER; $0-1400 \mathrm{~m}$

Thamniopsis langsdorffi (Hook.) W.R. Buck - ANG, ITA, MAG, MAN, NIT, NVF, PET, RJN, TER; 0$1400 \mathrm{~m}$

Thamniopsis pendula (Hook.) M. Fleisch. - RJN; s/alt; DD

Thamniopsis purpureophylla (Müll. Hal.) W.R. Buck - TER; ca. $1200 \mathrm{~m}$

Thamniopsis stenodictyon (Sehnem) Oliveira e Silva \& Yano - ANG, NIT; 200-1200 m

Thamniopsis undata (Hedw.) W.R. Buck - ITA, NVF, NVI, PET; 200-2300 m

Trachyxiphium aduncum (Mitt.) W.R. Buck - ITA, NVF, PET, TER; 900-2200 m

Trachyxiphium guadalupense (Brid.) W.R. Buck - NVF, RJN; 800-1400 m

Trachyxiphium hypnaceum (Müll. Hal.) W.R. Buck - PET; 0-1350 m

Trachyxiphium variable (Mitt.) W.R. Buck - RJN, TER; ?-1200 m

Plagiotheciaceae (1/2)

Plagiothecium lucidum (Hook f. \& Wilson) Paris - ITA; 1200-2890 m

Plagiothecium novogranatense (Hampe) Mitt. - ITA, NVF; 0-2000 m

\section{Polytrichaceae (6/17)}

Atrichum androgynum var. oerstedianum (Müll. Hal.) Nyholm - NVF, RJN; 0-800 m

Itatiella ulei (Broth.) G. L. Smith - ITA, PET; 2000-2890 m

Oligotrichum riedelianum (Mont.) Mitt. - ITA, NVF, RJN, TER; 800-1100 m

Pogonatum campylocarpon (Müll. Hal.) Mitt. - ITA, NVF, TER; 1000-2000 m

Pogonatum pensilvanicum (Hedw.) P. Beauv. - ANG, ITA, NIT, NVF, NVI, PET, RJN, TER; 0-2890 m 
Pogonatum perichaetiale subsp. oligodus (Müll. Hal.) Hyvönen - ITA; ca. 2000 m; VU

Pogonatum tortile (Sw.) Brid. - ITA, NVF; 800-1200 m

Polytrichadelphus magellanicus (Hedw.) Mitt. - MAG, NVF, RJN, TER; 0-2000 m

Polytrichadelphus pseudopolytrichum G. L. Smith. - TER; 500-1100 m

Polytrichadelphus semiangulatus (Brid.) Mitt. - ITA, MAG, NVF, PET, RJN, TER; 0-2500 m

Polytrichum angustifolium Mitt. - ITA; 900-2890 m

Polytrichum brasiliense Hampe - ANG, ITA, NIT, NVF; 200-2000 m

Polytrichum commune L. - ANG, ITA, MAG, NIT, NVF, PAR, PET, TER; 0-2100 m

Polytrichum glabrum Brid. - MAG, PET; ca. 1600 m; DD

Polytrichum juniperinum Willd.var. juniperinum - ANG, CAB, ITA, MAN, NIT, NVF, PAR, PET, RES, RJN, TER; 0-2500 m

Polytrichum juniperinum var. paulenese (Geh. \& Hampe) Herzog - ANG, MAN, NVF, PET, TER; 560$2200 \mathrm{~m}$

Polytrichum subcarinatum Hampe - S/L; ?-1000 m; DD

\section{Pottiaceae (17/39)}

Anoectangium aestivum (Hedw.) Mitt. - PET; 800-1200 m

Barbula indica (Hook.) Spreng. - ANG, NIT, RJN; 0-1200 m

Barbula lurida Hornsch. - RJN; 800-1200 m; DD

Barbula sambakiana Broth. - ITA; 0-1000 m; DD

Chenia leptophylla (Müll. Hal.) R.H. Zander - ANG, ITA, NIT, NVF, RJN; 0-1500 m

Didymodon amblyophyllus (Hook.) Broth. - S/L; s/alt; DD

Ganguleea angulosa (Broth. \& Dix.) R.H. Zander - ITA; 150-700 m

Hymenostomum fasciculatum Hampe - RJN; s/alt; DD

Hyophilla involuta (Hook.) A. Jaeger - ANG, CAB, MAN, NIT, RJN; 0-700 m

Hyophilla ochracea Broth. - ITA; 1000-1400 m; VU

Hyophilla ovalifolia (Hampe) Hampe - RJN; 0-1200 m; DD

Hyophilla regnelliii Müll. Hal. - PET, RJN; ?-1100 m; DD

Hyophilla rubiginosa Hampe - S/L; s/alt; DD

Hyophilla variegata Aongstr. - S/L; 0-1100 m

Hyophiladelphus agrarius (Hedw.) R.H. Zander - ACA, PAR, RJN, TER; 0-800 m

Leptodontium araucarieti (Müll. Hal.) Paris - ITA, NVF; 0-2500 m

Leptodontium filicola Herzog - ITA, RJN, TER; 1800-2500 m

Leptodontium flexifolium (Dicks.) Hampe - ITA; 2460 m; VU

Leptodontium stellatifolium (Hampe) Broth. - ITA; 2100-2931 m; VU

Leptodontium viticulosoides var. sulphureum (Müll. Hal.) R.H. Zander - ITA, RJN, TER; 0- $1850 \mathrm{~m}$

Leptodontium viticulosoides var. viticulosoides (P. Beauv.) Wijk \& Margad. - ITA, NVF, TER; 100$2200 \mathrm{~m}$

Leptodontium wallisii (Müll. Hal.) Kindb. - ITA; 1300-2750 m; VU

Plaubelia sprengelii (Schwägr.) R.H. Zander - ANG; nível do mar; VU

Pseudosymblefaris schimperiana (Paris) H.A. Crum - ITA; 1100-2300 m

Syntrichia amphidiacea (Müll. Hal.) R.H. Zander - TER; 700-1650 m

Syntrichia fragilis (J. Taylor) Ochyra - ITA; 600-2000 m

Timmiella barbuloides (Brid.) Moenk. - MAG, NIT, NVF, RJN; 0-1100 m; VU

Tortella humilis (Hedw.) Jenn. - ITA, MAG, NIT, NVI, PET, RJN, TER; 0-1400 m

Tortella linearis (Web. \& Mohr) R.H. Zander - RJN; s/alt; DD

Tortella tortuosa (Hedw.) Limpr. - ITA; 2000-2600 m; VU

Tortula muralis Hedw. - S/L; 0-900 m

Trichostomum prionodon Müll. Hal. - ITA; 1000-2000 m; DD

Trichostomum subcirrhatum Hampe - ITA; ca. 2100 m; DD

Trichostomum weisioides Müll. Hal. - ITA; 500-1500 m; VU

Weissia canaliculata Hampe - S/L; s/alt; DD

Weissia controversa Hedw. - RJN; 0-900 m 
Weissia glazioui R.H. Zander - S/L; 0-900 m

Weissia jamesonii J. Taylor - S/L; s/alt; DD

Weissia micacea (Schlencht.) Müll. Hal - NIT, RJN; 0-200 m; DD

Prionodontaceae (1/1)

Prionodon densus (Hedw.) Müll. Hal. - ITA, NVF, PET, RJN, TER; 400-2300 m

Pterobryaceae (8/10)

Calyptothecium duplicatum (Schwägr.) Broth. - MAN, NIT; 0-1100 m

Henicodium geniculatum (Mitt.) W.R. Buck - MIR, PET; 0-800 m

Jaegerina scariosa (Lorentz) Arz. - ANG, NIT; 0-1100 m

Orthostichidium subpendulum (Geh. \& Hampe) Broth. - RJN; 0-1200 m

Orthostichopsis tenuis (A. Jaeger) Broth. - RJN; 0-1100 m

Orthostichopsis tijucae (C.M.) Broth. - RJN; 0-800 m, VU

Orthostichopsis tortipilis (Müll. Hal.) Broth. - ITA, NVF, PET, RJN, TER; 0-1200 m

Pireella cymbifolia (Sull.) Cardot - MAN, NIT; nível do mar

Pterobryon densum (Schwägr.) Hornsch. - ANG, MAC, MAG, NIT, NVF, PET, RJN, TER; 600-1200 m

Spiridentopsis longissima (Raddi) Broth. - MAG, TER; 0-1000 m

Ptychomitriaceae (1/3)

Ptychomitrium patens (Müll. Hal.) Paris - ITA, TER; 0-1800 m

Ptychomitrium sellowianum (Müll. Hal.) A. Jaeger - NVF, PET, TER; 0-2200 m

Ptychomitrium vaginatum Besch. - NVF; 0-1200 m

Ptychomniaceae (1/1)

Ptychomnion fruticetorum Müll. Hal. - ITA; 900-1200 m

Racopilaceae (1/1)

Racopilum tomentosum (Hedw.) Brid. - ANG, CAB, ITA, MAG, MAN, NIT, NVF, NVI, PAR, PET, RES, RJN, SVJ, TER; 0-2700 m

\section{Rhabdoweisiaceae (1/1)}

Rhabdoweisia fugax (Hedw.) Bruch \& Schimp. - ITA; 2350-2890 m; DD

Rhacocarpaceae (1/3)

Rhacocarpus inermis var. cuspidatulus (Müll. Hal.) J.-P. Frahm - RJN, TER; ca. 800 m

Rhacocarpus inermis (Müll. Hal.) Lindb. var. inermis - ITA, NVF, PAR, PET, RJN, TER; 600-2800 m

Rhacocarpus purpurascens (Brid.) Paris - ITA, NVF, PET, TER; 800-2800 m

Rhyzogoniaceae (3/3)

Hymenodon aeruginosus (Hook f. \& Wilson) Müll. Hal. - ANG, ITA, MAN, NIT, RES, RJN, TER; 0$1000 \mathrm{~m}$

Pyrrhobryum spiniforme (Hedw.) Mitt. - ANG, ITA, NVF, RJN, TER, PAR, MAN, PET, MAG, NIT, RES, NVI; 0-2200 m

Rhizogonium novae-hollandiae (Brid.) Brid. - PET; 1100-1900 m; EN

Rigodiaceae (1/1)

Rigodium toxarion (Schwägr.) Schimp. - ITA, NVF, RJN, TER; 0-2300 m

Rutenbergiaceae (1/1)

Pseudocryphaea domingensis (Spreng.) W.R. Buck - ANG, NIT; 0-1300 m

Seligeriaceae $(2 / 2)$

Blindia magellanica Schimp. - ITA; 2200-2240 m; VU

Brachydontium notorogenes W.R. Buck \& Schäf.-Verw. - ITA; 2600-2700 m

Sematophyllaceae (10/48)

Acroporium catharinense Sehnem - NVF; 0-1100 m; VU

Rodriguésia 56 (87): 13-49. 2005 
Acroporium estrellae (Müll. Hal.) W.R. Buck \& Schäf.-Verw. - ITA, MAG, NVF, RJN, TER; 0-1900 m Acroporium exiguum (Broth.) W.R. Buck \& Schäf.-Verw. - PAR, TER; 730-900 m Acroporium longirostre (Brid.) W.R. Buck - ANG, MAN, NIT, RJN; 0-800 m Acroporium pungens (Hedw.) Broth. - ANG, CAB, ITA, NVF, PET, RJN, TER; 0-1200 m Aptychella proligera (Broth.) Herzog - ITA, NVF, NVI; 1650-2160 m Aptychopsis pyrrophylla (Müll. Hal.) Wijk \& Margad. - ITA; 0-2890 m Donnellia commutata (Müll. Hal.) W.R. Buck - ITA, RJN; 0-1350 m Donnellia lageniformis (Müll. Hal.) W.R. Buck - NVF; 200-2100 m Meiothecium boryanum (Müll. Hal.) Mitt. - CAB; nível do mar Rhaphidorrhynchium amoenum (Hedw.) M. Fleisch. - S/L; s/alt; DD Rhaphidorrhynchium distantifolium (Müll. Hal.) Broth. - RJN; s/alt; DD Rhaphidorrhynchium incurvum (Hampe) M. Fleisch. - RJN; ca. 800 m; DD Rhaphidorrhynchium lignicola (Aongstr.) Broth. - RJN; 800-1000 m; DD Rhaphidorrhynchium macrorhynchum (Hornsch.) Broth. - RJN, SVJ; 0-900 m Rhaphidorrhynchium olfersii (Hornsch.) Broth. - RJN; s/alt; DD

Rhaphidorrhynchium symbolax (Müll. Hal.) Broth. - ITA, MAG, NVF; 400-1600 m

Rhaphidorrhynchium tereticaule (Müll. Hal.) Broth. - ITA; s/alt; DD

Sematophyllum adnatum (Michx.) Brid. - ANG, MAN, NIT, NVF, RJN; 0-1300 m

Sematophyllum affine (Hornsch.) Mitt. - RJN; nível do mar; DD

Sematophyllum beyrichii (Hornsch.) Broth. - NVF; 0-1300 m

Sematophyllum campicola (Broth.) Broth. - NVF; 500-1000 m

Sematophyllum cyparissoides (Hornsch.) R.S. Williams - NVF, RJN, SVJ, TER; 0-2300 m

Sematophyllum decumbens Mitt. - TER; 800-1200 m; DD

Sematophyllum demissum (Wilson) Mitt. - ANG, NIT; 500-1110 m

Sematophyllum galipense (Müll. Hal.) Mitt. - CAB, RJN, TER; 0-800 m

Sematophyllum glaziovii (Hampe) O. Yano - ITA; 500-2750 m

Sematophyllum implanum Mitt. - RJN; nível do mar; DD

Sematophyllum leucostomum (Hampe) W.R. Buck - ITA, NVF; 0-1700 m

Sematophyllum lonchophyllum (Mont.) J. Flrosch. - MAG; nível do mar; DD

Sematophyllum minutum Broth. - RJN; 0-800 m

Sematophyllum oedophysidium W.R. Buck - S/L; s/alt; DD

Sematophyllum subdepressum (Hampe) Broth. - ITA, NVF, TER; 0-1200 m

Sematophyllum subfulvum (Broth.) Broth. - RJN; 0-900 m

Sematophyllum subpinnatum (Brid.) E. Britton - ACA, ANG, ARA, CAB, ITA, MAC, MAG, MAN, NIT, NVF, NVI, PAR, PET, RJN, ROS, SAQ, SVJ, TER; 0-1500 m

Sematophyllum subsecundum (A. Jaeger) Broth. - S/L; ?-900 m; DD

Sematophyllum subsimplex (Hedw.) Mitt. - ANG, CAB, ITA, MAN, NIT, NVF, PAR, RJN, SVJ, TER; 0$2000 \mathrm{~m}$

Sematophyllum succedaneum (Hook f. \& Wilson) Mitt. - NVF, RJN; 0-1300 m

Sematophyllum swartzii (Schwägr.) W.H. Welch \& H.A. Crum - ITA; 2400-2500 m; VU

Taxithelium planum (Brid.) Mitt. - ANG, CAB, MAN, NIT, PAR, SVJ, RJN; 0-1000 m

Trichosteleum glaziovii W.R. Buck - ITA, MAG, NVF, PET; 400-2300 m

Trichosteleum janeirense Broth. - S/L; s/alt; DD

Trichosteleum papillosissimum (Hampe) Broth. - S/L; 0-800 m

Trichosteleum papillosum (Hornsch.) A. Jaeger - NVF, TER; nível do mar

Trichosteleum pusillum (Hornsch.) A. Jaeger - RJN; s/alt; DD

Trichosteleum sentosum (Sull.) A. Jaeger - PAR; 0-1300 m

Trichosteleum subdemissum (Besch.) A. Jaeger - ANG, MAN, NIT, PAR, TER; 0-1200 m

Wijkia flagellifera (Broth.) H.A. Crum- PET; 0-1350 m

\section{Sphagnaceae (1/45)}

Sphagnum brevirameum Hampe - ITA, RJN; 800-2500 m

Sphagnum capillifolium var. capilifolium (Ehrhart) Hedw. - ITA, PET; 0-2200 m 
Sphagnum capillifolium var. tenerum (Sull. \& Lesq.) H.A. Crum - NVF; 1800-2400 m; DD Sphagnum costae var. confertorameum H.A. Crum \& Pinheiro da Costa - NVF; 1450 m; EN Sphagnum costae var. costae H.A. Crum \& Pinheiro da Costa - NVF; 1300 m; EN Sphagnum costae var. seriatum H.A. Crum \& Pinheiro da Costa - NVF; 1040 m; EN Sphagnum cuspidatum var. cuspidatum Ehrh. - CAF, ITA, RJN; 0-1200 m Sphagnum cuspidatum fo. serrulatum (Schlieph.) Pilous - ITA, PET; 1900-2500 m; DD Sphagnum cyclocladum Warnst. - RES; 800-1200 m; DD Sphagnum cyclophyllum Sull. \& Lesq. - RES; 600-1200 m Sphagnum exquisitum H.A. Crum - ITA; 2300 m; VU Sphagnum fontanum Müll. Hal. - RJN; 0-800 m; DD Sphagnum globicephalum Müll. Hal. - ITA; 1200-2500 m; DD Sphagnum gracilescens var. angustifrons Warnst. - RJN; 800-1200 m; DD Sphagnum gracilescens var. gracilescens Müll. Hal. - ITA, PET, RJN; 700-2750 m Sphagnum gracilescens var. laxifolium (Warnst.) Warnst. - PET, RJN; 800-1200 m; DD Sphagnum gracilescens var. submolluscum (Hampe) Warnst. - ITA, NVF, PET, RJN; 800-2500 m; DD Sphagnum guanabarae H.A. Crum - RJN; ca. 600 m; EN Sphagnum lindbergii Schimp. - ITA; 1900-2200 m; VU Sphagnum longicomosum Müll. Hal. - RJN; nível do mar; EN Sphagnum longistolo Müll. Hal. - ITA, NVF, RJN, TER; 1200-2500 m Sphagnum magellanicum Brid. - CAF, ITA, MAG, NVF, PAR, PET, RJN, TER; 0-2500 m Sphagnum meridense (Hampe) Müll. Hal. - ITA, NVF, PET, RJN, TER; 0-2500 m Sphagnum minutulum Müll. Hal. \& Warnst. - ITA; 2100 m; DD Sphagnum molle Sull. - S/L; s/alt; DD Sphagnum oxyphyllum Warnst. - ITA, TER; 0-2300 m Sphagnum palustre L. - ITA, MAC, NVF, RJN, TER; 0-1500 m Sphagnum papillosum Lindb. - ITA, RES; 300-1000 m Sphagnum perforatum Warnst. - ITA; 800-2100 m Sphagnum perichaetiale var. perichaetiale Hampe - ITA, MAC, PET, RES, RJN, TER; 0- $1400 \mathrm{~m}$ Sphagnum perichaetiale var. ramulosum Hampe - ITA, RJN, PET, TER; s/alt Sphagnum platyphylloides Warnst. - ITA; 800-2100 m; VU Sphagnum pseudoramulinum H.A. Crum - ITA; 2400-2500 m; VU Sphagnum recurvum P. Beauv. - ITA, NVF, PET, TER; 0-2200 m Sphagnum roseum Sull. - ITA; 2100-2500 m; VU Sphagnum rotundatum Müll. Hal. \& Warnst. - ITA, RES; 2000-2500 m; VU Sphagnum rotundifolium Müll. Hal. - ITA; 2100 m; DD Sphagnum sparsum Hampe - ITA; 1800-2500 m Sphagnum subovalifolium var. pumilum (Müll. Hal. \& Warnst.) Warnst. - ITA; 2400 m; DD Sphagnum subovalifolium var. subovalifolium Müll. Hal. \& Warnst. - ITA; 2300 m; DD Sphagnum subrufescens Warnst. - ITA; 2400 m; DD Sphagnum subsecundum Nees - ANG, ITA, NVF, NVI, RJN, TER; 0-1200 m Sphagnum sucrei H.A. Crum - NVF, RJN; 1000-2000 m; VU Sphagnum tenellum Ehrh. - PET; 2200 m; DD Sphagnum tenerum Sull. \& Lesq. - TER; 0-1200 m

Splachnaceae (2/2)

Tayloria arenaria (Müll. Hal.) Broth. - ITA; 1200-2000 m; DD

Tetraplodon itatiaiae Müll. Hal. - ITA; 800-2000 m; VU

\section{Stereophyllaceae (4/4)}

Entodontopsis leucostega (Brid.) W.R. Buck \& Ireland - NIT, RJN; 0-1000 m Eulacophyllum cultelliforme (Sull.) W.R. Buck \& Ireland - ITA, RJN, TER; 0-500 m Pilosium chlorophyllum (Hornsch.) Müll. Hal. - ANG, CAB, MAN, NIT, NVF, PET, SVJ; 0-800 m Stereophyllum radiculosum (Hook.) Mitt. - ACA, ANG, NIT, NVF, TER; 0-800 m 
Symphyodontaceae (1/1)

Symphyodon imbricatifolius (Mitt.) S.P. Churchill - CAB, ITA; 850-2500 m

Thuidiaceae (3/14)

Cyrto-hypnum involvens (Hedw.) W.R. Buck \& H.A. Crum - NVF; 0-800 m

Cyrto-hypnum minutulum (Hedw.) W.R. Buck \& H.A. Crum - NVF, NVI; 0-2000 m

Cyrto-hypnum schistocalyx (Müll. Hal.) W.R. Buck \& H.A. Crum - ITA, NVF; 0-1000 m

Thuidiopsis furfurosa (Hook f. \& Wilson) M. Fleisch. - ITA, PET, RJN, TER; 0-2000 m

Thuidium brasiliense Mitt. - NVF, PAR, TER; 0-1200 m

Thuidium delicatulum (Hedw.) Bruch \& Schimp. - ITA, NVF, PAR, RJN, TER; 0-2750 m

Thuidium granulatum (Hampe) A. Jaeger - S/L; s/alt; DD

Thuidium pseudoprotensum (Müll. Hal.) Mitt. - NVF, RJN, TER; 0-1600 m

Thuidium recognitum (Hedw.) Lindb. - NVF, PAR; 0-1400 m

Thuidium subpinnatum Broth. - RJN; ?-900 m; DD

Thuidium subtamariscinum (Hampe) Broth. - TER; ca. 1200 m; DD

Thuidium tamariscinum (Hedw.) B.S.G. - TER; 500-1200 m

Thuidium tomentosum Besch. - ANG, CAB, MAG, MAN; 0-1350 m

Thuidium urceolatum Lorentz - ITA, RJN, TER; 0-1400 m

\section{Divisão Marchantyophyta (30/100/333)}

Acrobolbaceae (1/1)

Tylimanthus laxus (Lehm. \& Lindenb.) Spruce - ITA; 100-2400 m

Adelanthaceae (1/2)

Adelanthus carabayensis (Mont.) Grolle - ITA; 1200-1600 m

Adelanthus decipiens (Hook.) Mitt. - ITA; 1800-2350 m

Aneuraceae (2/11)

Aneura pinguis (L.) Dumort. - ITA, RJN; 0-800 m; DD

Riccardia amazonica (Spruce) S.W. Arnell. - S/L; 0-2200 m

Riccardia cataractarum (Spruce) Schiffn. - NVF, RJN; 0-1800 m

Riccardia chamedryfolia (With.) Grolle - ANG, CAB, NIT, NVF, RJN; 250-1800 m

Riccardia digitiloba (Spruce) Pagán - CAB, ITA, NVI, RJN; 0-1400 m

Riccardia emarginata (Steph.) Hell - S/L; 0-1000 m; DD

Riccardia fucoidea (Sw.) Schiffn. - ITA, NVF, RJN; 0-1500 m

Riccardia glaziovii (Spruce) Meenks - ANG, ITA, NIT, RJN; 800-2400 m

Riccardia metzgeriiformis (Steph.) R.M. Schust. - ANG, CAB, MAN, NIT, RJN; 0-800 m; VU

Riccardia multifida (L.) S.F. Gray. - S/L; 0-1000 m; DD

Riccardia regnellii (Aongstr.) Hell - ANG, CAB, NIT; 0-1000 m

Arnelliaceae (2/2)

Southbya organensis Herzog - PET; 1900-2200 m; EN

Gongylanthus liebmanianus (Lindenb. \& Gottsche) Steph. - ITA; > 2000 m; VU

Aytoniaceae (1/1)

Plagiochasma rupestre (Forster) Steph. - S/L; 0-1100 m

Balantiopsidaceae (3/8)

Balantiopsis brasiliensis Steph. - ITA; 800-2500 m

Isotachis aubertii (Schwägr.) Mitt. - ANG, ITA, MAG, NIT, NVF, PET, TER; 100-2200 m

Isotachis inflata Steph. - ITA; 800-2500 m

Isotachis multiceps (Lindenb. \& Gottsche) Gottsche - ITA, PET, RJN, TER; 700-2600 m

Isotachis serrulata (Sw.) Gottsche - MAG, RJN; 0-1500 m

Neesioscyphus argillaceus (Nees) Grolle - ITA, PAR; 0-1000 m

Neesioscyphus carneus (Nees) Grolle - PET, RJN, TER; 500-1400 m; VU 
Neesioscyphus homophyllus (Nees) Grolle - PAR; 500-1500 m; VU

\section{Calypogeiaceae (2/7)}

Calypogeia grandistipula (Steph.) Steph. - ITA; 800-2000 m; VU

Calypogeia laxa Gottsche \& Lindenb. - ITA, RJN; 0-1300 m

Calypogeia lechleri (Steph.) Steph. - RJN; 0-800 m; EN

Calypogeia miquelii Mont. - ANG, CAB, NIT; 0-1000 m

Calypogeia peruviana Nees \& Mont. - RJN; 0-1400 m

Calypogeia uncinulatula Herzog - TER; 800-1200 m; VU

Mnioloma cyclostipa (Spruce) R.M. Schust. - ITA; 1300-1900 m; VU

\section{Cephaloziaceae (3/7)}

Anomoclada portoricensis (Hampe \& Gottsche) Váña - PET; 500-1000 m

Cephalozia crassifolia (Lindenb. \& Gottsche) Fulford. - ITA, TER; 400-2300 m

Cephalozia crossii Spruce - ITA; 1700-2300 m; VU

Odontoschisma brasiliense Steph. - RJN; 0-200 m; EN

Odontoschisma denudatum (Nees) Dumort. - ITA; 500-1500 m

Odontoschisma falcifolium Steph. - ITA; 0-1500 m

Odontoschisma longiflorum (J. Taylor) Steph. - ITA; 0-2000 m

\section{Cephaloziellaceae (4/6)}

Cephaloziella divaricata (Sm.) Schiffn. - ITA, MAC; 600-288 m

Cephaloziella granatensis (J.B. Jack) Fulford. - TER; 1000-2000 m; VU

Cephaloziopsis intertexta (Gottsche) R.M. Schust. - NVI, TER; 0-1400 m; VU

Cylindrocolea planifolia (Steph.) R.M. Schust. - ITA; 0-200 m

Cylindrocolea rhizantha (Mont.) R.M. Schust. - ACA, ARA, CAF, MAC, MAR, NVI, ROS, SAQ; 0$1000 \mathrm{~m}$

Kymatocalyx dominicensis (Spruce) Váña - ANG, CAB, ITA, MAN, NIT; 0-2300 m

\section{Chonecoleaceae (1/1)}

Chone colea doellingeri (Nees) Grolle - ACA, CAB, NIT, NVI, RJN; 0-1000 m

Fossombroniaceae (1/1)

Fossombronia porphyrorhiza (Nees) Prosk. - ANG, CAB; 0-1100 m

\section{Geocalycaceae (5/16)}

Clasmatocolea vermicularis (Lehm.) Grolle - ITA, NVF; 500-2400 m

Heteroscyphus combinatus (Nees) Schiffn. - TER; 0-1200 m; VU

Leptoscyphus amphibolius (Nees) Grolle - ITA, TER; 800-1200 m

Leptoscyphus gibbosus (J. Taylor) Mitt. - ITA; ca. 800 m; VU

Leptoscyphus porphyrius (Nees) Grolle - ITA, PET; 0-2200 m

Leptoscyphus spectabilis (Steph.) Grolle - ITA, PET; 800-2400 m

Lophocolea bidentata (L.) Dumort. - ANG, ITA, NVI; 0-1500 m

Lophocolea connata (Sw.) Nees - NIT, TER; 500-2000 m; VU

Lophocolea glaziovii Steph. - RJN; 0-800 m; DD

Lophocolea lindmannii Steph. - ITA, TER; 0-1500 m

Lophocolea mandonii Steph. - ITA; 1000-2500 m; VU

Lophocolea martiana subsp. bidentula (Nees) Gradst. - ANG, CAB, ITA, MAN, NIT, NVF, NVI, RJN, TER; $0-1850 \mathrm{~m}$

Lophocolea muricata (Lehm.) Nees - ITA, PET, TER; 250-2300 m

Lophocolea perissodonta (Spruce) Steph. - ITA; 0-1500 m

Lophocolea trapezoides Mont. - ITA; 400-1500 m

Saccogynidium caldense (Aongstr.) Grolle - ITA; 0-1600 m

\section{Gymnomitriaceae (2/2)}

Marsupella microphylla R.M. Schust. - ITA; 2300-2400 m; VU

Rodriguésia 56 (87): 13-49. 2005 
Stephaniella paraphyllina J.B. Jack - ITA; 2100-2500 m; VU

\section{Herbertaceae (2/8)}

Herbertus angustevittatus (Steph.) Fulford - ANG, NIT; 0-1100 m

Herbertus divergens (Steph.) Herzog - ANG, NIT, NVI; 0-900 m

Herbertus grossispinus (Steph.) Fulford - PET, TER; ca. 2000 m; VU

Herbertus juniperoideus (Sw.) Grolle - ITA; 2200-2300 m

Herbertus oblongifolius (Steph.) Gradst. \& Cleef - ITA; ca. 1800 m; VU

Herbertus pensilis (J. Taylor) Spruce - ITA; 1150-1800 m

Herbertus serratus Spruce - ITA, TER; 1000-2100 m

Triandrophyllum subtrifidum (Hook.f. \& J. Taylor) Fulford \& Hatch. - ITA; 2000-2500 m; VU

Jubulaceae (1/21)

Frullania apiculata (Reinw. et al.) Nees - MAC; 0-3000 m

Frullania arecae (Spreng.) Gottsche - ITA, MAN, NIT, NVF; 0-2000 m

Frullania atrata (Sw.) Nees - ITA, NVI; 500-2000 m

Frullania beyrichiana (Lehm. \& Lindenb.) Lehm. \& Lindenb. - ANG, CAB, MAN, NIT, RJN, TER; 0$1780 \mathrm{~m}$

Frullania brasiliensis Raddi - ANG, CAB, MAG, MAN, NIT, NVF, NVI, RJN, TER; 0-2200 m

Frullania caulisequa (Nees) Nees - ACA, ANG, CAB, CAF, ITA, MAC, MAN, MAR, NIT, QUI, ROS, SAQ, TER; 0-1000 m

Frullania dusenii Steph. - ACA, CAB, CAP, ITA, MAC, NIT; 0-2200 m

Frullania ecklonii (Spreng.) Gottsche et al. - ITA; 0-2400 m; VU

Frullania ericoides (Nees) Mont. - ACA, ANG, ARA, CAB, CAF, CAP, MAC, MAG, MAN, MAR, NIT, NVI, QUI, RJN, ROS, SJB, TER; 0-1300 m

Frullania gaudichaudii (Nees \& Mont.) Nees \& Mont. - RJN; 0-200 m; EN

Frullania gibbosa Nees - NIT, PET, RJN; 0-1200 m

Frullania glomerata (Lehm. \& Lindenb.) Mont. - ITA, MAC; 0-2400 m

Frullania intumescens (Lehm. \& Lindenb.) Lehm. \& Lindenb. - S/L; ca. 500-1000 m; VU

Frullania kunzei (Lehm. \& Lindenb.) Lehm. \& Lindenb. - ACA, ANG, ARA, CAB, CAF, CAP, MAC, MAN, MAR, NVI, QUI, ROS, SAQ; 0-2400 m

Frullania montagnei Gottsche - ANG, NIT; 0-1200 m

Frullania mucronata (Lehm. \& Lindenb.) Lehm. \& Lindenb. - ITA, RJN; 500-1200 m

Frullania riojaneirensis (Raddi) Aongstr. - ANG, ITA, MAN, NIT, RJN; 0-1100 m

Frullania schaefer-verwimpii Yuzawa \& Hatt. - TER; 0-1100 m; VU

Frullania setigera Steph. - ANG, ITA, NIT; 0-2000 m

Frullania supradecomposita (Lehm. \& Lindenb.) Lehm. \& Lindenb. - ANG, MAN, NIT, NVF, RJN; 0$1000 \mathrm{~m}$

Frullania vitalii Yuzawa \& Hatt. - MAC; 0-1000 m

\section{Jungermanniaceae (6/14)}

Anastrophyllum auritum (Lehm.) Steph. - ITA; 1300-2500 m; VU

Anastrophyllum piligerum (Nees) Steph. - ITA; 0-1550 m; VU

Anastrophyllum tubulosum (Nees) Grolle - ITA, PAR; 1000-2500 m

Cryptochila grandiflora (Lindenb. \& Gottsche) Grolle - ITA; 1750-2500 m; VU

Jamesoniella rubricaulis (Nees) Grolle - NVI, PAR; 500-2500 m

Jungermannia amoena Lindenb. \& Gottsche - ITA, PAR, PET, RJN, TER; 0-2500 m

Jungermannia hyalina Lyell - ITA; 500-2400 m; VU

Jungermannia sphaerocarpa Hook. - ITA; 2000-2500 m; VU

Lophozia bicrenata (Schmid.) Dumort. - ITA; ca. 2400 m; VU

Syzygiella anomala (Lindenb. \& Gottsche) Steph. - ITA; 1900-2200 m

Syzygiella integerrima Steph. - ITA; 1700-2300 m; VU

Syzygiella liberata Inoue - ITA; 1750-2280 m; VU

Syzygiella perfoliata (Sw.) Spruce - ITA, PAR, PET; 500-1950 m 
Syzygiella uleana Steph. - ITA, NVF; 1400-2000 m; VU

\section{Lejeuneaceae $(42 / 117)$}

Acanthocoleus aberrans (Lindenb. \& Gottsche) Kruijt - CAB; 50-2000 m

Acrolejeunea emergens (Mitt.) Steph. - ANG, CAB, MAC, NIT; 0-500 m

Acrolejeunea torulosa (Lehm. \& Lindenb.) Schiffn. - MAC; 0-800 m

Amphilejeunea reflexistipula (Lehm. \& Lindenb.) Gradst. - MAG; 100-1350 m

Anoplolejeunea conferta (Meissn.) A. Evans - NVF, NVI; 0-2400 m

Aphanolejeunea asperrima Steph. - ITA; 1400-2000 m; VU

Aphanolejeunea camillii (Lehm.) R.M. Schust. - ITA; 0-1600 m

Aphanolejeunea gracilis Jovet-Ast - ITA; 50-1900 m

Aphanolejeunea microscopica var. africana (Pócs) Pócs \& Bernecker -SMM, TER; 1080-1920m

Aphanolejeunea paucifolia (Spruce) E. Reiner - MAC, RJN; 0-1350 m; VU

Aphanolejeunea sintenisii (Steph.) Steph. - ITA; 1100-1400 m; VU

Aphanolejeunea truncatifolia Horik - ITA, MAC, MAN, QUI, SJB, TER; 0-1400 m

Archilejeunea parviflora (Nees) Schiffn. - ANG, CAF, MAC, NIT, ROS; 0-1500 m

Aureolejeunea fulva R.M. Schust. - ITA; 2200-2600 m; VU

Blepharolejeunea incongrua (Lindenb. \& Gottsche) Van Slageren \& Kruijt - ITA; 1650-2600 m; VU

Blepharolejeunea securifolia (Steph.) R.M. Schust. - ITA; >2000 m; VU

Brachiolejeunea laxifolia (J. Taylor) Schiffn. - ITA, NVF; 1000-2500 m

Brachiolejeunea phyllorhiza (Nees) Kruijt \& Gradst. - ITA, NVF; 300-1500 m

Bromeliophila natans (Steph.) R.M. Schust. - MAC; nível do mar; EN

Bryopteris diffusa (Sw.) Nees - ANG, CAB, MAG, MAN, NIT, NVF, PET, RJN, TER; 0- $1500 \mathrm{~m}$

Bryopteris filicina (Sw.) Nees - ANG, MAN, NIT, NVI, PET, RJN, TER; 0-2000 m

Caudalejeunea lehmanniana (Gottsche) A. Evans - ANG, MAN, NIT; 0-500 m

Ceratolejeunea ceratantha (Nees \& Mont.) Steph. - S/L; 0-900 m

Ceratolejeunea cornuta (Lindenb.) Schiffn. - PAR; 0-1000 m

Ceratolejeunea cubensis (Mont.) Schiffn. - ANG, CAB, NIT, NVI; 0-500 m

Ceratolejeunea fallax (Lehm. \& Lindenb.) Bonner - ITA, MAG, NVF, RJN; 0-1600 m

Ceratolejeunea rubiginosa Gottsche - ANG, CAB, MAN, NIT, SVJ; 40-400 m

Cheilolejeunea acutangula (Nees) Grolle - ITA, RJN, TER; 0-2300 m

Cheilolejeunea clausa (Nees \& Mont.) R.M. Schust. - MAC, QUI; 0-800 m

Cheilolejeunea discoidea (Lehm. \& Lindenb.) Kachr. \& R.N. Schust. - ITA; 0-2400 m

Cheilolejeunea holostipa (Spruce) R.-L. Zhu \& Grolle - ITA, RJN; 0-2200 m

Cheilolejeunea inflexa (Hampe) Grolle - ITA, RJN; 2000-2600 m; EN

Cheilolejeunea insecta Grolle \& Gradst. - ITA; 1100-2450 m

Cheilolejeunea oncophylla (Aongstr.) Grolle \& E. Reiner - ITA; 300-2000 m

Cheilolejeunea rigidula (Mont.) R.M. Schust. - ANG, ARA, CAB, CAF, MAC, MAN, NIT, QUI ROS, SAQ; 0-1000 m

Cheilolejeunea trifaria (Reinw. et al.) Mizut - ANG, CAB, MAN, NIT; 0-1000 m

Cololejeunea cardiocarpa (Mont.) A. Evans - ACA, MAC, NIT, SAQ; 0-1000 m

Cololejeunea minutissima (Sm.) Schiffn. - ACA, MAC; 0-1500 m

Cololejeunea obliqua (Nees \& Mont.) Schiffn. - ANG, MAC, NIT; 0-300 m

Cololejeunea subcardiocarpa Tixier - ITA; 0-2000 m

Colura calyptrifolia (Hook.) Dumort. - ITA; 2350-2400 m; VU

Colura itatyana Steph. - ITA; 2300-2400 m; VU

Colura tenuicornis (A. Evans) Steph. - TER; 0-2000 m

Colura ulei Jovet-Ast - ANG; 0-200 m

Diplasiolejeunea alata Jovet-Ast - ITA; 700-1500 m

Diplasiolejeunea brunnea Steph. - ANG, NIT, RJN; 0-800 m

Diplasiolejeunea pauckertii (Nees) Steph. - ITA; 1500-2300 m; VU

Diplasiolejeunea pellucida (Meissn.) Schiffn. - ANG, NIT, RJN; 0-1000 m

Diplasiolejeunea replicata (Spruce) Steph. - ITA; 0-2300 m 
Diplasiolejeunea rudolphiana Steph. - PET; 0-800 m

Diplasiolejeunea unidentata (Lehm. \& Lindenb.) Schiffn. - TER; ca. 1000 m; VU

Drepanolejeunea aculeata Bischl. - RJN; 0-1000 m; EN

Drepanolejeunea anoplantha (Spruce) Steph. - NVI; 0-2000 m

Drepanolejeunea araucariae Steph. - ITA; 700-2000 m

Drepanolejeune a campanulata (Spruce) Steph. - ITA, RJN; 700-2000 m

Drepanolejeunea fragilis Bischl. - NVI; 0-1000 m

Drepanolejeunea granatensis (J.B. Jack \& Steph.) Bischl. - ITA; >2000 m; VU

Drepanolejeunea inchoata (Meissn.) Schiffn. - RJN; 1000-2000 m; DD

Drepanolejeunea lichenicola (Spruce) Steph. - TER; 500-2000 m

Drepanolejeunea mosenii (Steph.) Bischl. - ANG, ITA, MAC, NIT, RJN; 0-2000 m

Drepanolejeunea orthophylla (Nees \& Mont.) Bischl. - ANG, ITA, MAN, NIT, RJN; 0-900 m

Drepanolejeunea palmifolia (Nees) Steph. - TER; 0-500 m

Frullanoides corticalis (Lehm. \& Lindenb.) Van Slageren - MAG; 0-500 m

Frullanoides densifolia Raddi - ITA, MAG, RJN; 0-2000 m

Harpalejeunea oxyphylla (Nees \& Mont.) Steph. - ANG, MAN; 0-1000 m

Harpalejeunea schiffneri S.W. Arnell - ITA, NVI, TER; 0-1800 m

Harpalejeunea subacuta A. Evans - ITA; 2000-2400 m; VU

Lejeunea anomala Lindenb. \& Gottsche - RJN; 0-1100 m; DD

Lejeunea bermudiana (A.Evans) R.M. Schust. - CAB, MAN, NIT; 0-200 m

Lejeunea caespitosa Lindenb. - ANG, CAB, MAN, MAR, NIT, NVI; 0-800 m

Lejeunea capensis Gottsche - ITA, PAR; 1400-1900 m

Lejeune a cerina (Lehm. \& Lindenb.) Gottsche - TER; 0-1300 m

Lejeune a cristulata (Steph.) E. Reiner \& Goda - ITA, MAC, QUI; 900-1500 m

Lejeunea flava (Sw.) Nees - ANG, ARA, CAB, ITA, MAC, MAN, MAR, NIT, NVI, RJN, SAQ; 0-2400 m

Lejeunea glaucescens Gottsche - ANG, CAB, MAC, MAN, NIT, TER; 0-1100 m

Lejeunea grossiretis (Steph.) E. Reiner \& Goda - PET, TER; >500 m; EN

Lejeunea grossitexta (Steph.) E. Reiner \& Goda - ITA, NVI, TER; 0-1500 m

Lejeunea laeta (Lehm. \& Lindenb.) Gottsche - NVI; 0-900 m

Lejeune laetevirens Nees \& Mont. - ANG, CAB, MAC, MAN, NIT, NVI, SAQ; 0-1500 m

Lejeunea lepida Lindenb. \& Gottsche - S/L; 0-800 m; DD

Lejeunea monimiae (Steph.) Steph. - ITA; 0-2000 m

Lejeunea phyllobola Nees \& Mont. - ACA, ANG, ARA, CAF, CAP, MAC, MAR, NIT, NVI, ROS, SAQ; $0-250 \mathrm{~m}$

Lejeunea raddiana Lindenb. - NVI; 0-500 m

Lejeunea trinitensis Lindenb. - ANG, MAC, MAN, NIT; 0-800 m

Lepidolejeunea eluta (Nees) R.M. Schust. - S/L; 100-1050 m

Leptolejeunea brasiliensis Bischl. - RJN; 0-1000 m

Leptolejeunea elliptica (Lehm. \& Lindenb.) Schiffn. - ANG, ITA, NIT, RJN; 0-1500 m

Leptolejeunea exocellata (Spruce) A. Evans. - ITA; 100-1500 m

Leptolejeunea maculata (Mitt.) Schiffn. - S/L; 0-200 m; DD

Leptolejeunea moniliata Steph. - ANG, MAN, NIT; 0-1000 m

Leucolejeunea unciloba (Lindenb.) A. Evans - ANG, CAB, CAP, MAC, NIT, NVF; 0-1300 m

Leucolejeunea xanthocarpa (Lehm. \& Lindenb.) A. Evans - ANG, CAB, ITA, MAC, MAR, NIT, NVI, NVF; $0-2500 \mathrm{~m}$

Lopholejeunea nigricans (Lindenb.) Schiffn. - ANG, CAB, NIT; 0-1000 m

Lopholejeunea subfusca (Nees) Schiffn. - ANG, CAB, ITA, MAC, MAN, NIT; 0-750 m

Marchesinia brachiata (Sw.) Schiffn. - ANG, CAB, ITA, MAN, NIT, NVF, NVI, PAR, RJN, TER; 0-1700 m

Metalejeunea cucullata (Reinw. et al.) Grolle - SVJ; 0-1350 m

Microlejeunea bullata (J. Taylor) Steph. - ACA, ITA, MAC, NIT, QUI, SAQ, SJB; 0-2400 m

Microlejeunea subulistipa Steph. - ITA; 0-2000 m; EN

Myriocoleopsis gymnocolea (Spruce) E. Reiner \& Gradst. - S/L; 100-1300 m 
Neurolejeunea breutelii (Gottsche) A. Evans - ITA, NVI; 0-1850 m Odontolejeunea decemdentata (Spruce) Steph. - DQC; 0-1150 m; VU

Odontolejeunea lunulata (Weber) Schiffn. - S/L; 0-1800 m

Omphalanthus filiformis (Sw.) Nees - ANG, ITA, NIT, NVF, RJN, TER; 150-2000 m

Pluvianthus squarrosus (Steph.) R.M. Schust. \& Schäf.-Verw. - ITA; 500-2350 m

Prionolejeunea aemula (Gottsche) A. Evans. - ITA, NVI; 0-1200 m

Prionolejeunea denticulata (Weber) Schiffn. - RJN; 0-200 m; DD

Pycnolejeunea densistipula (Lehm. \& Lindenb.) Steph. - S/L; 0-1000 m; DD

Rectolejeunea berteroana (Gottsche) A. Evans - NVI; 0-1000 m

Schiffneriolejeunea polycarpa (Nees) Gradst. - ACA, ANG, ITA, MAG, MAN, NIT, NVI, RJN, TER; 0$1000 \mathrm{~m}$

Stictolejeunea squamata (Willd.) Schiffn. - ANG, ITA, NIT; 0-1500 m

Symbiezidium barbiflorum (Lindenb. \& Gottsche) A. Evans - ANG, MAC, RJN; 0-1500 m

Symbiezidium transversale (Sw.) Trevis. - NIT; 0-1000 m

Taxilejeunea isocalycina (Ness) Steph. - ITA, NVI; 0-800 m

Taxilejeunea lusoria (Lindenb. \& Gottsche) Steph. - ITA; 0-2300 m

Taxilejeunea pterigonia (Lehm. \& Lindenb.) Schiffn. - ITA; 0-1500 m

Vitalianthus bischlerianus (Pôrto \& Grolle) R.M. Schust. \& Giancotti. - ITA; 0-1800 m

Xylolejeunea crenata (Nees \& Mont.) X.-L. He \& Grolle. - S/L; 0-1000 m

Lepidoziaceae (7/22)

Arachniopsis diacantha (Mont.) Howe - ANG, NIT, NVI, RJN, TER; 0-1000 m

Bazzania cuneistipula (Gottsche \& Lindenb.) Trevis. - ITA; 1200-2000 m; VU

Bazzania gracilis (Hampe \& Gottsche) Steph. - RJN; 0-1500 m

Bazzania heterostipa (Steph.) Fulford. - PET; 0-1800 m

Bazzania hookeri (Lindenb.) Trevis. - ITA, NVI, PET; 0-2000 m

Bazzania jamaicensis (Lehm. \& Lindenb.) Trevis - ITA; 800-1400 m

Bazzania longistipula (Lindenb.) Trevis. - ITA; 400-2400 m

Bazzania nitida (Weber) Grolle - TER; 0-1780 m

Bazzania pallide-virens (Steph.) Fulford. - ANG, NIT; 0-800 m

Bazzania schlimiana (Gottsche) Fulford. - ITA; 800-2000 m; VU

Bazzania stolonifera (Sw.) Trevis. - ANG, NIT, TER; 0-1600 m

Bazzania taleana (Gottsche) Fulford. - ITA; 800-2100 m; VU

Kurzia brasiliensis (Steph.) Grolle - ANG, NIT, NVF; 400-2000 m

Kurzia capillaris (Sw.) Grolle - ANG, ITA, NIT, NVF, PET; 100-2500 m

Kurzia flagellifera (Steph.) Grolle - ITA, ca. 1000 m; VU

Lepidozia coilophylla J. Taylor - RJN; 0-1000 m

Lepidozia cupressina (Sw.) Lindenb. - ITA, RJN; 800-2400 m

Lepidozia inaequalis (Lehm. \& Lindenb.) Lehm. \& Lindenb. - ANG, NIT, NVF; 100-2100 m

Micropterygium pterygophyllum (Nees) Trevis. - TER; 0-500 m

Paracromastigum dusenii (Steph.) R.M. Schust. - ITA; 2350-2400 m; VU

Paracromastigum pachyrhizum (Nees) Fulford - ITA; 500-2400 m

Telaranea nematodes (Gottsche) M.A. Howe - ANG, ITA, MAC, NIT; 0-2400 m

Lunulariaceae (1/1)

Lunularia cruciata (L.) Dumort. - RJN; 0-1250 m

Marchantiaceae (2/7)

Dumortiera hirsuta (Sw.) Nees - ANG, CAB, ITA, MAN, NIT, NVI, RJN, TER; 0-2000 m

Marchantia berteroana Lehm. \& Lindenb. - ANG, NIT; 0-2300 m

Marchantia breviloba A. Evans - ITA, PAR; 900-1350 m; VU

Marchantia chenopoda L. - ANG, ITA, MAN, NIT, NVI, PET TER; 0-1500 m

Marchantia paleacea Bert. - S/L; s/alt; DD 
Marchantia papillata Raddi - ANG, CAB, NIT, NVF, RJN; 0-1000 m

Marchantia polymorpha L. - S/L; 0-1000 m

\section{Metzgeriaceae (1/20)}

Metzgeria agnewiae Kuwah. - ITA; 800-2100 m

Metzgeria albinea var. albinea Spruce - ANG, CAB, ITA, MAN, NIT, NVF, PAR, SVJ, TER; 0-1800 m Metzgeria albinea var. angusta (Steph.) Costa \& Gradst. - ANG, MAN, NVF, RJN; 0-1000 m

Metzgeria aurantiaca Steph. - ANG, ITA, MAN, NIT, NVF, PAR, RJN, SVJ; 0-1600 m

Metzgeria brasiliensis Schiffn. - ITA, NVF, NVI, PAR, RJN, SVJ; 0-1200 m

Metzgeria conjugata Lindb. - ITA, NVF; 0-2000 m

Metzgeria convoluta Steph. - ANG, ITA, MAN, NIT, NVF, RJN, TER; 0-1200 m

Metzgeria cratoneura Schiffn. - ITA, TER; 0-1100 m

Metzgeria decipiens (C. Massal.) Schiffn. \& Gottsche - NVI, RJN; 0-2500 m

Metzgeria dichotoma (Sw.) Nees - ITA, MAN, NIT, NVF, RJN; 0-1600 m

Metzgeria fruticola Spruce - ITA, NVF; 800-1100 m

Metzgeria furcata (L.) Dumort. - CAB, MAN, NIT, NVF, NVI, RJN, TER; 0-1500 m

Metzgeria herminieri Schiffner - ITA, NVF, PET; 0-2000 m

Metzgeria lechleri Steph. - ITA, PAR, RJN, TER; 0-2500 m

Metzgeria leptoneura Spruce - ITA, NVF, TER; 0-2500 m

Metzgeria myriopoda Lindb. - ANG, CAB, NVF, PAR, TER; 0-2000 m

Metzgeria psilocraspeda Schiffn. - ITA; 0-2000 m

Metzgeria rufula Spruce - ITA, RJN; 100-800 m

Metzgeria scyphigera A. Evans - ITA; 800-2100 m

Metzgeria subaneura Schiffn. - PAR; 100-2500 m

\section{Monocleaceae (1/1)}

Monoclea gottschei subsp. elongata Gradst. \& Mues - ANG, CAB, ITA, MAN, NIT, NVF, NVI, PAR, $\mathrm{RJN} ; 0-2000 \mathrm{~m}$

\section{Pallaviciniaceae (3/6)}

Jensenia erythropus (Gottsche) Grolle - ITA; ca. 2000 m; EN

Pallavicinia lyellii (Hook.) S.F. Gray - RJN; 100-1000-2000 m (?)

Symphyogyna aspera Steph. - ANG, CAB, ITA, MAN, NIT, PAR, RJN; 0-2200 m

Symphyogyna brasiliensis (Nees) Nees \& Mont. - ANG, CAB, ITA, MAN, NIT, NVF, NVI, PAR, RJN, TER; 0-1850 m

Symphyogyna brongniartii Mont. - ITA, RJN; 0-1000 m

Symphyogyna podophylla (Thunb.) Mont. \& Nees. - ITA, NIT, NVF, NVI, PET; 500-1800 m

Pelliaceae (1/1)

Noteroclada confluens J. Taylor - ITA, NVI, TER; 400-2500 m

\section{Plagiochilaceae (1/22)}

Plagiochila adiantoides (Sw.) Lindenb. - ITA; 1000-2200 m

Plagiochila bifaria (Sw.) Lindenb. - ITA; 0-2200 m

Plagiochila boryana Gottsche - PET; 1900 m (?); EN

Plagiochila corrugata (Nees) Nees \& Mont. - ITA, TER; 0-2300 m

Plagiochila cristata (Sw.) Lindenb. - ITA, NVI, TER; 700-1500 m

Plagiochila disticha (Lehm. \& Lindenb.) Lindenb. - NVI; 0-900 m

Plagiochila distinctifolia Lindenb. - ANG, NIT; 0-1000 m

Plagiochila diversifolia Lindenb. \& Gottsche - TER; ca. 1300-2000 m; VU

Plagiochila exigua (J. Taylor) J. Taylor - ITA; 1500-2400 m; VU

Plagiochila flaccida Lindenb. - ITA; 2100-2300 m; DD

Plagiochila gymnocalycina (Lehm. \& Lindenb.) Lindenb.- ITA, NVF, NVI, PAR, TER; 500-2400 m

Plagiochila lingua Steph. - S/L; 0-800 m; DD 
Plagiochila macrostachya Lindenb. - ITA; 1500-2200 m; VU

Plagiochila martiana (Nees) Lindenb. - ANG, MAC, MAN, NIT, NVI, PET; 0-1100 m

Plagiochila micropteryx Gottsche - ITA; 0-1000 m

Plagiochila montagnei Nees - ANG, CAB, MAN, NIT; 0-1200 m

Plagiochila patentissima Lindenb. - CAB, ITA, NVI; 0-1400 m

Plagiochila patula (Sw.) Lindenb. - ITA, NVI; 900 m; VU

Plagiochila raddiana Lindenb. - S/L; 0-1350 m

Plagiochila rutilans Lindenb. - ANG, ITA, MAN, NIT; 0-2400 m

Plagiochila simplex (Sw.) Lindenb. - ANG, MAN, PET, TER; 0-1700 m

Plagiochila subplana Lindenb. - ANG, ITA, MAN, NIT; 0-1300 m

\section{Porellaceae (1/2)}

Porella brasiliensis (Raddi) Schiffn. - ANG, ITA, MAG, MAN, NIT, PET, TER; 0-1500 m

Porella reflexa (Lehm. \& Lindenb.) Trevis. - MAG; 0-1500 m

\section{Radulaceae (1/18)}

Radula angulata Steph. - ITA; 0-1950 m

Radula fendleri Gottsche - ITA; 1000-1700 m; VU

Radula gottscheana J. Taylor - ITA; 0-1200 m; VU

Radula javanica Gottsche - ANG, MAN; 0-1650 m

Radula kegelii Gottsche-CAB, MAG; 0-1350 m

Radula ligula Steph. - ANG, RJN; 0-800 m

Radula mexicana Lindenb. \& Gottsche - ANG, ITA, NIT; 0-1100 m

Radula nudicaulis Steph. - ITA, NVF; 800-2700 m

Radula obovata Castle. - ITA, MAG; 0-1000 m

Radula pocsii K. Yamada. - ITA; ca. 1450 m; VU

Radula quadrata Gottsche - ITA; 100-2000 m

Radula recubans J. Taylor - ITA, NVI, PET; 800-1000 m

Radula schaefer-verwimpii K. Yamada - ITA; 900-2300 m; VU

Radula sinuata Gottsche - S/L; 500-2000 m

Radula stenocalyx Mont. - S/L; 0-2300 m

Radula tectiloba Steph. - ITA; 0-1650 m

Radula tenera Mitt. - ITA, NVI; 500-2200 m

Radula voluta J. Taylor - S/L; 500-2400 m

\section{Ricciaceae (1/5)}

Riccia curtisii (James) Austin. - S/L; 0-500 m

Riccia grandis Nees - S/L; nível do mar; DD

Riccia plano-biconvexa Steph. - NIT; 0-650 m

Riccia stenophylla Spruce - NIT; 0-1000 m

Riccia wainionis Steph. - S/L; 0-1200 m

\section{Scapaniaceae (1/1)}

Scapania portoricensis Hampe \& Gottsche - ITA, NVF, NVI; 800-2500 m

Trichocoleaceae (1/2)

Trichocolea brevifissa Steph. - ITA, NVF; 100-2500 m

Trichocolea flaccida (Spruce) J.B. Jack \& Steph. - ANG, NIT; 800-1700 m 


\section{Agradecimentos}

A primeira autora agradece ao Conselho Nacional de Desenvolvimento Científico e Tecnológico ( $\mathrm{CNPq})$, pelas duas bolsas de Iniciação Científica concedidas e que foram fundamentais para o desenvolvimento deste trabalho. Os autores também agradecem aos doutores Allan Fife (Manaaki Whenua Landcare Research), Bruce Allen e Richard Zander (Missouri Botanical Garden), Ronald Pursell (The Pensnsylvania State University), que gentilmente checaram a taxonomia de espécies dos seus grupos de estudo, bem como a Prof. Anna Olga de Barros Barreto pela correção da versão do resumo em inglês.

\section{REFERÊNCIAS BIBLIOGRÁFICAS}

Allen, B. H. 1987. A revision of the genus Leucomium (Leucomiaceae). Memoirs of the New York Botanical Garden 45: 661677.

Bastos, C. J. P. 1999. Briófitas de restinga das regiões metropolitana de Salvador e litoral norte do estado da Bahia, Brasil. Dissertação. Universidade de São Paulo. $173 \mathrm{p}$.

Bastos, C. J. P.; Albertos, B. \& Villas-BôasBastos, S. B. 1998a. Bryophytes from some Caatinga areas in the state of Bahia (Brazil). Tropical Bryology 14: 69-75.

Bastos, C. J. P.; Stradmann, M. T. S. \& VillasBôas-Bastos, S. B. 1998b. Additional Contribution to the Bryophyte Flora of Chapada Diamantina National Park, State of Bahia, Brazil. Tropical Bryology 15: 15-20.

Bastos, C. J. P. \& Villas-Bôas-Bastos, S. B. 1998. Adições à brioflora (Bryopsida) do Estado da Bahia, Brasil. Tropical Bryology 15: 111-116.

Bastos, C. J. P.; Yano, O. \& Villas-BôasBastos, S. B. 2000. Briófitas de campos rupestres da Chapada Diamantina, Estado da Bahia, Brasil. Revista Brasileira de Botânica 23: 357-368.

Brotherus, V. F. 1924. Ergebnisse der botanischen Expedition der Kaiserlichen
Akademie der Wissenschaften nach Südbrasilien 1901, heralsgegeben von Prof. Dr. V. Schiffner. Denkschr. Akademie der Wissenschaften in Wien 83: 251-358.

Buck, W. R. 1979. A re-evaluation of the Bruchiaceae with the description of a new genus. Brittonia 31 (4): 469-473.

Buck, W. R. 1998. Pleurocarpous Mosses of the West Indies. Memoirs of the New York Botanical Garden 1: 1-401.

Buck, W. R. \& Ireland, R. R. 1989. Plagiotheciaceae. Flora Neotropica 50: 1-22.

Câmara, P. E. A. S. 2002. Levantamento da brioflora das matas de galeria da reserva ecológica do IBGE, RECOR, Distrito Federal. Dissertação. Universidade de Brasília. $140 \mathrm{p}$.

Castro, N. M. C. F. 1997. Bryopsida do Parque Nacional de Sete Cidades, Piauí, Brasil. Dissertação. Universidade Federal de Pernambuco. 85 p.

Churchill, S. P. \& Linares C., E. L. 1995a. Prodromus bryologiae Novo-Granatensis: introducción a la flora de musgos de Colombia. Parte 1: Adelotheciaceae a Funariaceae. Biblioteca Jose Jeronimo Triana 12: 1-453.

1995b. Prodromus bryologiae NovoGranatensis: introducción a la flora de musgos de Colombia. Parte 2: Grimmiaceae a Trachypodaceae. Biblioteca Jose Jeronimo Triana 12: 455-924.

1998. Catalog of Amazonian Mosses. The Journal of the Hattori Botanical Laboratory 85: 191-238.

Costa, D. P. 1988. Leucobryaceae do Parque Nacional da Tijuca no Estado do Rio de Janeiro (Brasil). Rodriguésia 64/66 (41/ 40): 41-48.

1992. Hepáticas do Pico da Caledônea. Nova Friburgo, Rio de Janeiro, Brasil. Acta Botanica Brasilica 6 (1): 3-39. 1994. Musgos do Pico da Caledônea, município de Nova Friburgo, estado do Rio de Janeiro, Brasil. Acta Botanica Brasilica 8 (2): 141-191. 
1997. Bryophyta e Hepatophyta. In: Marques, M.C.M. (org.) Mapeamento da cobertura vegetal e listagem das espécies ocorrentes na Área de Proteção Ambiental de Cairuçu, município de Parati, RJ. Série Estudos e Contribuições 13: 1-96.

1999. Metzgeriaceae (Metzgeriales, Hepatophyta) no Brasil. Tese. Universidade de São Paulo. Instituto de Biociências, São Paulo. 261 p.

2003. Floristic composition and diversity of Amazonian rainforest bryophytes in Acre, Brazil. Acta Amazonica 33 (3): 399-414.

Costa, D. P.; Imbassahy, C. A. A. \& Silva, V. P. A. V. 2005. Cheklist and distribution of the mosses, liverworts and hornworts of the Rio de Janeiro state, Brazil. The Journal of the Hattori Botanical Laboratory (no prelo).

Costa, D. P.; Imbassahy, C. A. A. \& Silva, V. P. A. V. Status de conservação das espécies de briófitas do estado do Rio de Janeiro (inédito).

Costa, D. P. \& Lima, F. M. 2005. Moss diversity in the tropical rainforests of Rio de Janeiro, Southeastern Brazil. Revista Brasileira de Botânica (no prelo).

Costa, D. P. \& Silva, A. G. 2003. Briófitas da Reserva Natural da Vale do Rio do Doce, Linhares, Espírito Santo, Brasil. Boletim do Museu de Biologia Mello Leitão 16: 21-38.

Costa, D. P. \& Yano, O. 1988. Hepáticas talosas do Parque Nacional da Tijuca, Rio de Janeiro, Brasil. Acta Botanica Brasilica 1 (2): 73-82.

Costa, D. P. \& Yano, O. 1995. Musgos do município de Nova Friburgo, Rio de Janeiro, Brasil. Arquivos do Jardim Botânico do Rio de Janeiro 33 (1): 99-118.

Costa, D. P. \& Yano, O. 1998. Briófitas da restinga de Macaé, Rio de Janeiro, Brasil. Hoehnea 25: 99-119.

Crosby, M. R., Magill, R. E., Allen, B. \& He, S. 1999. A Checklist of the Mosses. Missouri Botanical Garden. 325 p.(http://
www.mobot.org/MOBOT/tropicos/most/ checklist.shtml).

Crum, H. A. 1990a. Comments on Sphagnum sect. Sphagnum in South America. Contributions from the University of Michigan Herbarium 17: 71-81. 1990b. A new look at Sphagnum sect. Acutifolia in South America. Contributions from the University of Michigan Herbarium 17: 83-91.

1990c. Preliminary notes on Sphagnum sect. Subsecunda in South America. Contributions from the University of Michigan Herbarium 17: 93-97. 1992. Miscellaneous Notes of the Genus Sphagnum. 3. New Species from Brazil. The Bryologist 95 (4): 419-429.

1993. Progress toward understanding Sphagnum section Sphagnum in Brazil. Advances in Bryology 5: 9-29.

Delgadillo, M. C.; Bello, B. \& Cárdenas, S. M. A. 1995. LATMOSS: A Catalogue of Neotropical Mosses (http://www.mobot.org/ MOBOT/tropicos/most/latmoss.shtml).

Dusén, P. 1903. Sur la flore de la Serra do Itatiaya au Brésil. Arquivos do Museu Nacional do Rio de Janeiro 13: 1-119.

Fife, A. J. 1987. Taxonomic and nomenclatural observations on the Funariaceae 5. A revision of the Andean species of Entosthodon. Memoirs of the New York Botanical Garden 45: 301-325.

Florschütz-de-Waard, J. 1996. Musci. Part. III. In: Görts-van Rijn, A.R.A. (ed.), Flora of the Guianas. Royal Botanic Gardens, Kew, pp. 384-438.

Frahm, J.-P. 1991. Dicranaceae: Campylopodioideae, Paraleucobryoideae. Flora Neotropica 54: 1-238.

1996. Revision der Gattung Rhacocarpus Lindb. (Musci). Cryptogamie: Bryologie, Lichénologie 17:39-65.

. 1997. A taxonomic revision of Dicranodontium (Musci). Annales Botanici Fennici 34: 179-204.

Fundação S.O.S. Mata Atlântica. 2002. Atlas da evolução dos remanescentes florestais 
e ecossistemas associados do domínio da mata atlântica no período 1995-2000. São Paulo, Fundação S.O.S. Mata Atlântica/ INPE.

Germano, S. R. 2003. Florística e ecologia das comunidades de briófitas de umremanescente de floresta atlântica (Reserva ecológica do Gurjaú, Pernambuco, Brasil). Tese. Universidade Federal de Pernambuco. 126 p.

Gradstein, S. R. 1995. Diversity of Hepaticae and Anthocerotae in montane forests of the tropical Andes. In: Churchill, S.P. et al. (eds.). Biodiversity and Conservation of Neotropical Montane Forests. New York Botanical Graden. p. 321-334.

Gradstein, S. R.; Churchill, S. P. \& SalazarAllen, N. 2001. Guide to the Bryophytes of Tropical America. Memoirs of the New York Botanical Garden 86: 1-577.

Gradstein, S. R. \& Costa, D. P. 2003. The Hepaticae and Anthocerotae of Brazil. Memoirs of the New York Botanical Garden 87: 1-336.

Gradstein, S. R.\& Pócs, T. 1989. Bryophytes. In: Lieth, H. \& Werger, M.J.A. (eds.). Tropical Rain Forest Ecosystems. Elsevier Science Publishers. Amsterdam, pp. 311325.

Hallingbäck, T. \& Hodgetts, N. 2000. Mosses, liverworts \& hornworts: a status survey and conservation action plan for bryophytes. IUCN, Gland. 106 p.

Hallingbäck, T.; Hodgetts, N. \& Urmi, E. 1996. How to use the new IUCN Red List categories on bryophytes. Guidelines proposed by the IUCN SSC Bryophyte Specialist Group. Anales del Instituto de Biologia de la Universidad Nacional Autónoma de México, Serie Botánica 67(1): 47-157.

Hampe, E. 1870. Musci frondosi. In: E. Warming (ed.). Symbolae ad floram Brasiliae centrales cognoscendam. Videnskabelige Meddelelser fra dansk naturhistoriske Forening i Kjöbenhavn, ser. 3, 8(10-20): 267-296.
1872. Musci frondosi. In: E. Warming (ed.). Symbolae ad floram Brasiliae centrales cognoscendam. Videnskabelige Meddelelser fra dansk naturhistoriske Forening i Kjöbenhavn, ser. 3, 10: 36-59. 1874a. Musci frondosi. In: E. Warming (ed.). Symbolae ad floram Brasiliae centrales cognoscendam. Videnskabelige Meddelelser fra dansk naturhistoriske Forening i Kjöbenhavn, ser. 3, 19 (9-11): 129-178.

1874b. Musci frondosi. In: E. Warming (ed.). Symbolae ad floram Brasiliae centrales cognoscendam. Videnskabelige Meddelelser fra dansk naturhistoriske Forening i Kjöbenhavn, ser. 3, 19 (12-16): 73-141.

1877. Musci frondosi. In: E. Warming (ed.). Symbolae ad floram Brasiliae centrales cognoscendam. Videnskabelige Meddelelser fra dansk naturhistoriske Forening i Kjöbenhavn, ser. 3, 24: 251-274. 1879. Enumeratio muscorum hactenus in provinciis Brasiliensibus Rio de Janeiro et São Paulo detectorum. Videnskabelige Meddelelser fra dansk naturhistoriske Forening i Kjöbenhavn 26: 73-164.

Hedenäs, L. 2003. Amblystegiaceae. Flora Neotropica 89: 1-107.

Heinrichs. J.; Anton, H.; Gradstein, S. R. \& Mues, R. 2000. Systematics of Plagiochila sect. Glaucescens Carl (Hepaticae) from tropical America: a morphological and chemotaxonomical approach. Plant Systematics and Evolution 220: $115-138$.

Herzog, T. 1925. Neue Bryophyten aus Brasilien. Repertorium specierum novarum regni vegetabilis 21: 22-38.

Hornschuch, C. F. 1840. Musci. In: Martius (ed.). Flora brasiliensis enumeratio plantarum in Brasilia hactenus detectarum quas suis aliorumque botanicorum studiis descriptas et methodo naturali digestas partim icone illustratas. 1(2): 1-712, pl. 1-82 (Bryophyta, 1-100, pl. 1-5). Monachii. 
Index of Mosses Database (W33OST) http:/ /mobot.mobot.org/W3 T/Search/ most.html.

Ireland, R. R. \& Buck, W. R. 1994. Stereophyllaceae. Flora Neotropica 65: 1-49.

LaFarge-England, C. 1998. The infrageneric phylogeny, classification and phytogeography of Leucoloma (Dicranaceae, Bryopsida). The Bryologist 101: 181-220.

Lemos-Michel, E. 1999. Briófitas epífitas sobre Araucaria angustifolia (Bert.) Kunze no Rio Grande do Sul, Brasil. Tese. Universidade de São Paulo, Instituto de Biociências, São Paulo. 318p.

Lisboa, R. C. L. \& Ilkiu-Borges, F. 1995. Diversidade das briófitas de Belém (PA) e seu potencial como indicadoras de poluição urbana. Boletim do Museu Paraense Emílio Goeldi, série Botânica 11: 199-225.

Lisboa, R. C. L. \& Ilkiu-Borges, F. 1997. Novas ocorrências de Bryophyta (musgos) para o estado do Pará, Brasil. Acta Amazonica 27 (2): 81-102.

Lisboa, R. C. L. \& Ilkiu-Borges, F. 2001. Briófitas de São Luís do Tapajós, município de Itaituba, com novas adições para o estado do Pará. Boletim do Museu Paraense Emílio Goeldi, série Botânica 17 (1): 75-91.

Lisboa, R. C. L.; Lima, M. J. L. \& Maciel, U. N. 1999. Musgos da ilha de Marajó - II município de Anajás Pará, Brasil. Acta Amazonica 29 (2): 201-206.

Molinaro, L. C. \& Costa, D. P. 2001. Briófitas do arboreto do Jardim Botânico do Rio de Janeiro. Rodriguésia 52: 107-124.

Müller, C. 1898. Bryologia Serrae Itatiaiae. Bulletin of the Herbier Boissier 6: 18-48. 1900. Symbolae ad bryologiam Brasiliae et regionum vicinarum. Hedwigia 39: 235-289.

1901. Symbolae ad bryologiam Brasiliae et regionum vicinarum. Hedwigia 40:55-99.

Muñoz, J. 1999. A revision of Grimmia (Musci, Grimmiaceae) in the Americas. Latin
America. Annals of the Missouri Botanical Garden 86: 118-191.

Ochi, H. 1980. A revision of the neotropical Bryoïdeae, Musci (First part). The Journal of the Faculty of Education, Tottori University, Natural Science 29: 49-154.

Ochi, H. 1981a. Taxonomic position of Anomobryopsis, Musci. Hikobia, Suppl. 1:55-57.

1981b. A revision of the neotropical Bryoïdeae, Musci (second part). The Journal of the Faculty of Education, Tottori University, Natural Science 30: 21-55.

1982. A revision of the Bryoiddeae (Musci) in southern South America). The Journal of the Faculty of Education, Tottori University, Natutal Science 31: 11-47.

Oliveira e Silva, M. I. M. N. 1998. Briófitas da Reserva Ecológica de Rio das Pedras, município de Mangaratiba, do Parque Estadual da Ilha Grande e da Reserva Biológica Estadual da Praia do Sul, município de Angra dos Reis, estado do Rio de Janeiro. Tese. Instituto de Biociências, Universidade de São Paulo. 321p.

Oliveira e Silva, M. I. M. N.; Milanez, A.I. \& Yano, O. 2002. Aspectos ecológicos de briófitas em áreas preservadas de mata atlântica, Rio de Janeiro, Brasil. Tropical Bryology 22: 77-102.

Oliveira e Silva, M. I. M. N. \& Yano, O. 1998. Ocorrências novas de briófitas para o Brasil. Revista Brasileira de Botânica 21: 125-134.

Pôrto, K. C. \& Bezerra, M. F. A. 1996. Briófitas da caatinga 2. Agrestina, Pernambuco. Acta Botanica Brasilica 18: 93-102.

Pôrto, K. C. \& Germano, S. R. 2002. Biodiversidade e importância das briófitas na conservação dos ecossistemas naturais de Pernambuco. In: Tabarelli, M. \& Silva, J. M. C. Diagnóstico da biodiversidade de Pernambuco. Recife: Massangana. 125-152.

Projeto Flora do Estado do Rio de Janeiro: bases para o uso sustentável da diversidade vegetal. 2002 (inédito). 
Pursell, R. A. 1994. Taxonomic notes on Neotropical Fissidens. The Bryologist 97: 253-271.

Reese, W. D. 1993. Calymperaceae. Flora Neotropica 58: 1-102.

Reiner-Drehwald, M. E. \& Goda, A. 2000. Revision of the genus Crossotolejeunea (Lejeuneaceae, Hepaticae). The Journal of the Hattori Botanical Laboratory 89: $1-54$.

Sá, P. S. A. 1995. Aspectos florísticos e ecológicos das briófitas do riacho Coité, Timbaúba-PE. Dissertação. Universidade Federal de Pernambuco, Recife. 59 p.

Santiago, R. L. 1997. Estudos brioflorísticos de três formações vegetais no município de Bonfim-Roraima. Dissertação. Universidade Federal de Pernambuco, Recife. $124 \mathrm{p}$.

Santos, R. C. P. \& Lisboa, R. C. L. 2003. Musgos (Bryophyta) do nordeste paraense, Brasil - 1. Zona Bragantina, microrregião do Salgado e município de Viseu. Acta Amazonica 33 (3): 415422.

Sastre-de-Jesus, I. 1987. A revision of the Neckeraceae Schimp. and Thamnobryaceae Margad. \& Dur. in the Neotropics. Dissertation, City University of New York.

Schäfer-Verwimp, A. 1989. New or interesting records of Brazilian bryophytes, II. The Journal of the Hattori Botanical Laboratory 67: 313-321.

1991. Contribution to the knowledge of the bryophyte flora of Espírito Santo, Brazil. The Journal of the Hattori Botanical Laboratory 69: 147-170.

1992. New or interesting records of Brazilian bryophytes, III. The Journal of the Hattori Botanical Laboratory 71: 55-68.

. 1996. New or interesting records of Brazilian bryophytes, V. Candollea 51: 283-302.

Schäfer-Verwimp, A. \& Giancotti, C. 1993. New or interesting records of Brazilian bryophytes, IV. Hikobia 11: 285-292.
Schäfer-Verwimp, A. \& Vital, D.M. 1989. New or interesting records of Brazilian bryophytes. The Journal of the Hattori Botanical Laboratory 66: 255-261.

Schultze-Motel, W. 1970. Monographie der Laubmoosgattung Andreaea. 1. Die costaten Arten. Willdenowia 6: 25-110.

Sehnem, A. 1969. Musgos Sul-Brasileiros. Pesquisas, Botânica 27: 1-36. 1970. Musgos Sul-Brasileiros II.

Pesquisas, Botânica 28: 1-117. 1972. Musgos Sul-Brasileiros III. Pesquisas, Botânica 29: 1-70. 1976. Musgos Sul-Brasileiros IV. Pesquisas, Botânica 30: 1-79. 1978. Musgos Sul-Brasileiros V. Pesquisas, Botânica 32: 1-170. 1979. Musgos Sul-Brasileiros VI. Pesquisas, Botânica 33: 1-149. 1980. Musgos Sul-Brasileiros VII. Pesquisas, Botânica 34: 1-121.

Sharp, A. J., Crum, H. A. \& Eckel, P. M. 1994. The Moss Flora of Mexico. Memoirs of the New York Botanical Garden 69: 11113.

Shaw, A. J. \& Goffinet, B. 2000. Bryophyte Biology. Cambridge University Press, England. 476p.

Spence, J. R. 1996. Rosulabryum genus novum (Bryaceae). The Bryologist 99: 221-225.

Stephani, F. 1905-1909. Species Hepaticarum 3: 1-693. Genève. 1909-1912. Species Hepaticarum 4: 1824. Genève.

Tixier, P. 1988. Le genre Glossadelphus Fleisch. (Sematophyllaceae, Musci) et sa valeur. Nova Hedwigia 46 (3-4): 319-356.

Uribe, J. \& Gradstein, S.R. 1999. Estado del conocimiento de la flora de hepáticas de Colombia. Revista de la Academia Colombiana de Ciencias Exactas, Físicas y Naturales 23 (87): 315-318.

Veloso, H. P., Rangel Filho, A. L. R. \& Lima, J. C. A. 1991. Classificação da Vegetação Brasileira adaptada a um Sistema 
Universal. IBGE/CDDI. Departamento de Documentação e Biblioteca, 123 p.

Villas-Bôas-Bastos, S. B. \& Bastos, C. J. P. 1998. Briófitas de uma área de cerrado no município de Alagoinhas, Bahia, Brasil. Tropical Bryology 15: 101-110.

Visnadi, S. R. 1998. Briófitas em ecosistemas costeiros do Núcleo Picinguaba do Parque Estadual da Serra do Mar, Ubatuba-SP. Tese. Universidade Estadual Paulista, São Paulo. 274 p. \& Vital, D. M. 2000. Lista de briófitas ocorrentes no parque estadual das fontes do Ipiranga-PEFI. Hoehnea 27 (3): 279-294. \& Vital, D. M. 2001. Briófitas das Ilhas de Alcatrazes, do Bom Abrigo, da Casca e do Castilho, Estado de São Paulo, Brasil. Acta Botanica Brasilica 15 (2): 255-270.

Vital, D. M. \& Visnadi, S. R. 1994. Bryophytes of Rio Branco Municipality, Acre, Brazil. Tropical Bryology 9: 69-74.

Yano, O. 1981. A checklist of Brazilian mosses. The Journal of the Hattori Botanical Laboratory 50: 279-456. 1984. Checklist of Brazilian liverworts and hornworts. The Journal of the Hattori Botanical Laboratory 56: 481-548.

1989. An additional checklist of Brazilian bryophytes. The Journal of the Hattori Botanical Laboratory 66: 371-434. 1995. A new additional annotated checklist of Brazilian bryophytes. The
Journal of the Hattori Botanical Laboratory 78: 137-182.

1996a. A checklist of Brazilian bryophytes. Boletim do Instituto de Botânica de São Paulo 10: 47-232. 1996b. Criptógamos do Parque Estadual dasFontes do Ipiranga, SãoPaulo, SP. Briófitas, 1: Mniaceae, Rhizogoniaceae, Racopilaceae, Phyllogoniaceae e Leucobryaceae (Bryales). Hoehnea 23(2): 81-98.

Yano, O. \& Colletes, A. G. 2000. Briófitas do Parque Nacional de Sete Quedas, Guaíra, PR, Brasil. Acta Botanica Brasilica 14: 215-242.

Yano, O. \& Costa, D. P. 2000. Flora dos estados de Goiás e Tocantins. Criptógamas: Briófitas. Vol. 5. Editora da Universidade Federal de Goiás. 33 p.

Yano, O. \& Mello, Z. R. 1999. Frullaniaceae dos manguezais do litoral sul de São Paulo. Iheringia, Botanica 52: 65-87.

Yano, O. \& Oliveira e Silva, M. I. M. N. 1997. Criptógamos do Parque Estadual das Fontes do Ipiranga, São Paulo, SP. Briófitas, 2: Fissidentaceae (Bryales). Hoehnea 24(2): 107-114.

Zander, R. H. 1972. Revision of the genus Leptodontium (Musci) in the New World. The Bryologist 75(3): 213-280.

Zander, R. H. 1993. Genera of the Pottiaceae: mosses of harsh environments. Bulletin of the Buffalo Society of Natural Sciences 32: 1-378. 

